NOTICE WARNING CONCERNING COPYRIGHT RESTRICTIONS:

The copyright law of the United States (title 17, U.S. Code) governs the making of photocopies or other reproductions of copyrighted material. Any copying of this document without permission of its author may be prohibited by law. 


\title{
Space Profiling for Parallel Functional Programs
}

\author{
Daniel Spoonhower $\quad$ Guy E. Blelloch Phillip B. Gibbons
Robert Harper \\ April 30, 2008 \\ CMU-CS-08-110,
}

School of Computer Science

Carnegie Mellon University

Pittsburgh, PA 15213

\begin{abstract}
This paper presents a semantic space profiler for parallel functional programs. Building on previous work in sequential profiling, our tools help programmers to relate runtime resource use back to program source code. Unlike many profiling tools, our profiler is based on a cost semantics. This provides a means to reason about performance without requiring a detailed understanding of the compiler or runtime system. It also provides a specification for language implementers. This is critical in that it enables us to separate cleanly the performance of the application from that of the language implementation.

Some aspects of the implementation can have significant effects on performance. Our cost semantics enables programmers to understand the impact of different scheduling policies yet abstracts away from many of the details of their implementations. We show applications where the choice of scheduling policy has asymptotic effects on space use. We explain these use patterns through a demonstration of our tools. We also validate our methodology by observing similar performance in our implementation of a parallel extension of Standard ML.
\end{abstract}


Keywords: parallelism, profiling, cost semantics, Standard ML $\because \because \ldots$ 


\section{Introduction}

Functional programming languages have been promoted as an attractive means for writing parallel programs. They offer many opportunities for parallel evaluation without requiring programmers to be explicit about concurrency. With a clear semantic definition, programmers can reason about the results of program evaluation independently of any particular implementation or target machine.

In reality, achieving better performance through parallelism can be quite difficult. While the extensional behavior of parallel functional programs does not depend on the language implementation, their performance certainly does. In fact, even sequential implementations of functional languages can have dramatic and unexpected effects on performance. To analyze and improve performance, functional programmers often rely upon profilers to analyze resource use [Appel et al., 1988, Runciman and Wakeling, 1993a, Sansom and Peyton Jones, 1995, Röjemo and Runciman, 1996]. With parallel implementations, the need for profilers is magnified by such issues as task granularity, communication, and scheduling policy-all of which can have a significant impact on time and space use.

In this paper, we present a semantic space profiler for a call-by-value parallel functional language and relevant to shared memory architectures. Our tools are the first to allow programmers to reason about the space use of parallel functional programs. Our method abstracts away from details of language implementation and yet allows programmers to reason about asymptotic performance. Because it is based on a semantics rather than a particular implementation, our profiling method remains true to the spirit of functional programming: 'thinking about program behavior does not require a detailed understanding of the compiler or target machine.

Our profiling method must account, at least abstractly, for some parts of the implementation. In this work, we focus on scheduling policy and its effects on application space use. Because the choice of scheduling policy often has dramatic, and even asymptotic, effects on space use (as detailed in this paper), it is critical that a programmer has the flexibility to choose a policy that is best-suited to his or her application. This flexibility must be reflected both in the language implementation and in any profiling tools.

Our profiling tools are based on a cost semantics [Sansom and Peyton Jones, 1995, Blelloch and Greiner, 1996]. A cost semantics is a dynamic semantics that, in addition to the ordinary extensional result, yields an abstract measure of cost. In our semantics, this cost is a pair of directed graphs that capture essential dependencies during program execution (Section 3). These graphs are used by our tools to simulate the behavior of different scheduling policies and to make predictions about space use. For example, by generating graphs for a range of inputs, programmers can perform an asymptotic analysis of space use. Our profiling tools also allow programmers to visualize the parallel execution of programs and compare scheduling policies (Section 5).

We emphasize that our method allows users to profile parallel programs. This stands in contrast to many existing profilers, which only provide a means of profiling a program based on a particular implementation. While this leads to some loss of precision, there is a tradeoff between precision and offering performance results that can be easily related to the program text. Our cost semantics is the fulcrum that allows us to balance this tradeoff.

Our cost semantics also provides a formal specification that forces language implementations to be "safefor-space" [Shao and Appel, 1994]. Besides acting as a guide for implementers, it maintains a clean separation between the performance of a program and the performance of the language implementation. This ensures that profiling results are meaningful and that programmers can expect the same asymptotic performance when moving from one compliant implementation to another.

To demonstrate that this specification does not place an onerous burden on implementers, we present an implementation of a parallel extension of Standard ML [Milner et al., 1997] based on our cost semantics (Section 6). Our framework also extends to other parallel extensions of ML (e.g., Fluet et al. [2007]) as well as languages with eager parallelism such as NESL [Blelloch et al., 1994] and Nepal [Chakravarty and Keller, 2000]. One advantage of our framework is that by factoring out scheduling, we can bring to light performance issues in languages such as NESL that bake in a particular scheduling policy.

Our implementation includes three different scheduling policies. As we also anticipate the need for other policies, we have isolated the core decisions of such policies behind a simple signature. 
We implemented several parallel algorithms to validate our work and measured performance using both our tools and by sampling memory use in our implementation. The results show that our cost semantics is able to correctly predict asymptotic trends in memory use (Section 7).

In summary, the contributions of our work include:

- the first space profiling tools for parallel functional programs,

- the first cost semantics supporting an analysis of space use under different scheduling policies,

- several formal implementations of our language whose space use provably matches the specification given by the cost semantics, and

- an extensible implementation in MLton [Weeks, 2006], a high-performance compiler and runtime system.

In the course of our implementation, we also discovered a space leak in one of the optimizations in MLton. As a specification, a cost semantics determines exactly which performance problems must be blamed on the programmer and which can be attributed to the language implementation.

\section{Motivating Example}

In the next section, we introduce a profiling semantics that assigns a space cost to each program. This cost abstracts away from many details of the compiler, but enables programmers to predict (within a constant factor) the space behavior of different scheduling policies. To motivate this work, we present a small example (matrix multiplication), where the choice of scheduling policy has a dramatic effect on space use. We give a cursory discussion here, and consider this application in further detail in Section 7, along with three other applications (sorting, convex hull, and $n$-body simulation).

Matrix multiplication offers a tremendous amount of potential parallelism. For inputs of length and width $n$, each of the $n^{3}$ scalar multiplications may be computed in parallel. (Recall that there are $n$ scalar multiplications for each of the $n^{2}$ elements in the result.) Figure 1 depicts the code written in our parallel extension of ML. The function reduce (not shown) aggregates data in parallel. Note that recursive calls to loop (shown as $\{\ldots\}$ ) in the definition of tabulate may also be computed in parallel.

Our framework can be used by programmers to predict the behavior of parallel programs such as matrix multiplication, as summarized below. In general, a programmer would:

1. Select a program and run it using the profiling interpreter based on our cost semantics.

The cost semantics yields a pair of directed graphs. As these graphs are too detailed to present in their raw form, our tools summarize these graphs into a more compact form. An example of summarized graphs for matrix multiplication is shown in Figure 2(a). In these graphs, nodes represent sequential computation. In the top graph, edges point downward, and an edge from $n_{1}$ to $n_{2}$ indicates that $n_{1}$ must be executed before $n_{2}$. For matrix multiplication, we see the regular structure of its parallelism: work is evenly distributed among parallel branches. In the bottom graph, edges point upward, and an edge from $n_{2}$ to $n_{1}$ indicates that $n_{2}$ depends on the value allocated at $n_{1}$.

At the first stage of analysis, these graphs allow programmers to make qualitative statements about their programs and to classify parallel algorithms visually: algorithms with different parallel structure will give rise to graphs with different shapes. These graphs are also used as input in the following step.

2. Use our simulator to predict the space performance for different scheduling policies and numbers of processors.

Each scheduling policy determines a traversal of the cost graphs. By fixing a policy and the number of processors, our simulator uses these graphs to determine the high-water mark for space use. It also determines the point during execution at which this mark is reached, as well as where in the source code this data is allocated and used. 


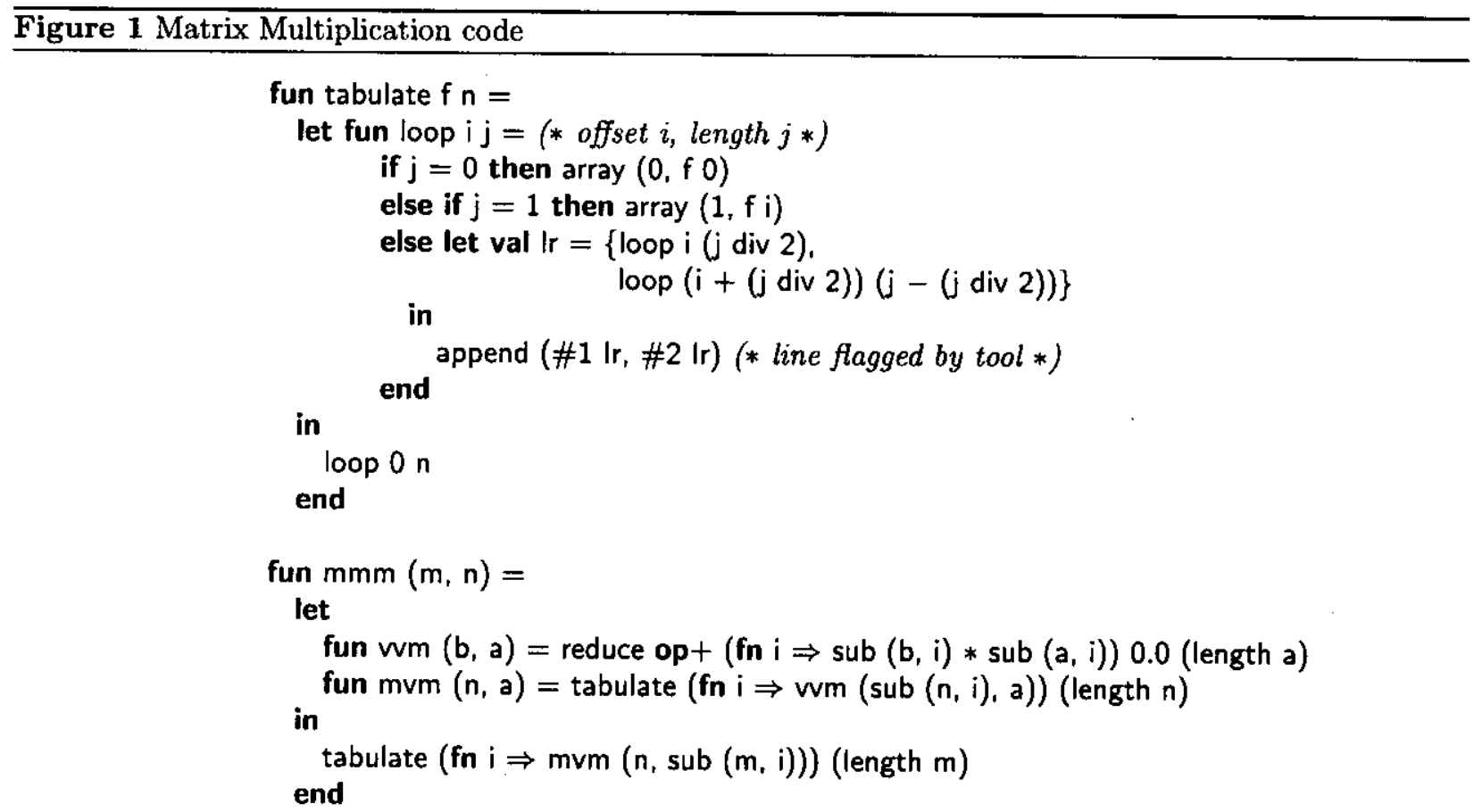

3. Repeat steps 1 and 2 for different inputs. Plot the results to draw conclusions about asymptotic performance.

For each input, programmers generate a new pair of graphs. Our tool can then be used to generate plots such as those shown in Figure 2(b). These plots show trends in space use as a function of input size for different schedulers. In this example, we compare two schedulers each using two processors. The scheduling policy on the left manages parallel tasks using a FIFO queue and implements a breadth-first traversal of the top cost graph. The scheduling policy on the right implements a parallel depth-first traversal (see Section 6.2 for details) of the top cost graph. Our tools also help explain the space use through a breakdown according to particular allocation points (as shown in the figure) or use points. As the figure shows, for both schedulers, a significant part of the space use at the high-water mark can be attributed to the arrays allocated in the implementation of tabulate (i.e., append $(\ldots)$, as marked in Figure 1). However, for the breadth-first scheduler (on the left), most of the space is attributed to the work queue and two forms of closure (denoted with "[...]" in the key). These two closures appear during the application of reduce.

4. Reexamine the cost graphs to isolate space use and elucidate the effects of the scheduling policy.

While the plots generated in the previous step depict trends in space use, they provide little insight into how the scheduling policy affects these trends. The final step in an analysis often requires programmers to revisit the cost graphs, this time including information about the scheduling policy. In the course of analyzing our example applications, we will show how computing the difference between two executions based on different schedules can explain why one policy yields better performance and how programs can be modified to improve performance. In our matrix multiplication example (bottom graph of Figure 2(a)), we see that the point where the graph is the widest (i.e., where the most parallelism is available) also marks a shift in how the progxam uses space. From this point on, most of the data allocated by the program are no longer in use. Our tools can show that the high-water mark for space under a breadth-first policy arises because all these nodes at the widest point are concurrently active. 
Figure 2 Cost Graphs and Simulated Results for Matrix Multiplication. This figure shows (a) summarized cost graphs and (b) space use as a function of input size for two scheduling policies: breadth-first (left) and depth-first (right).

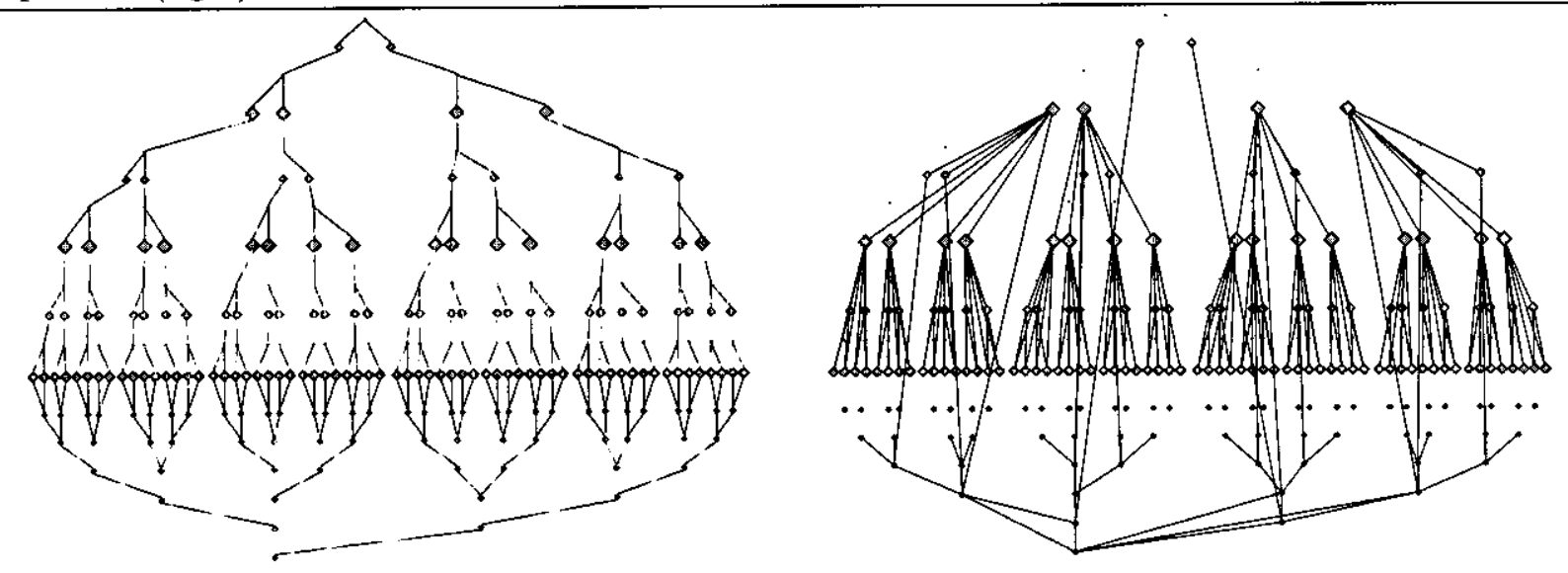

(a) summarized cost graphs for $4 \times 4$ matrix multiplication
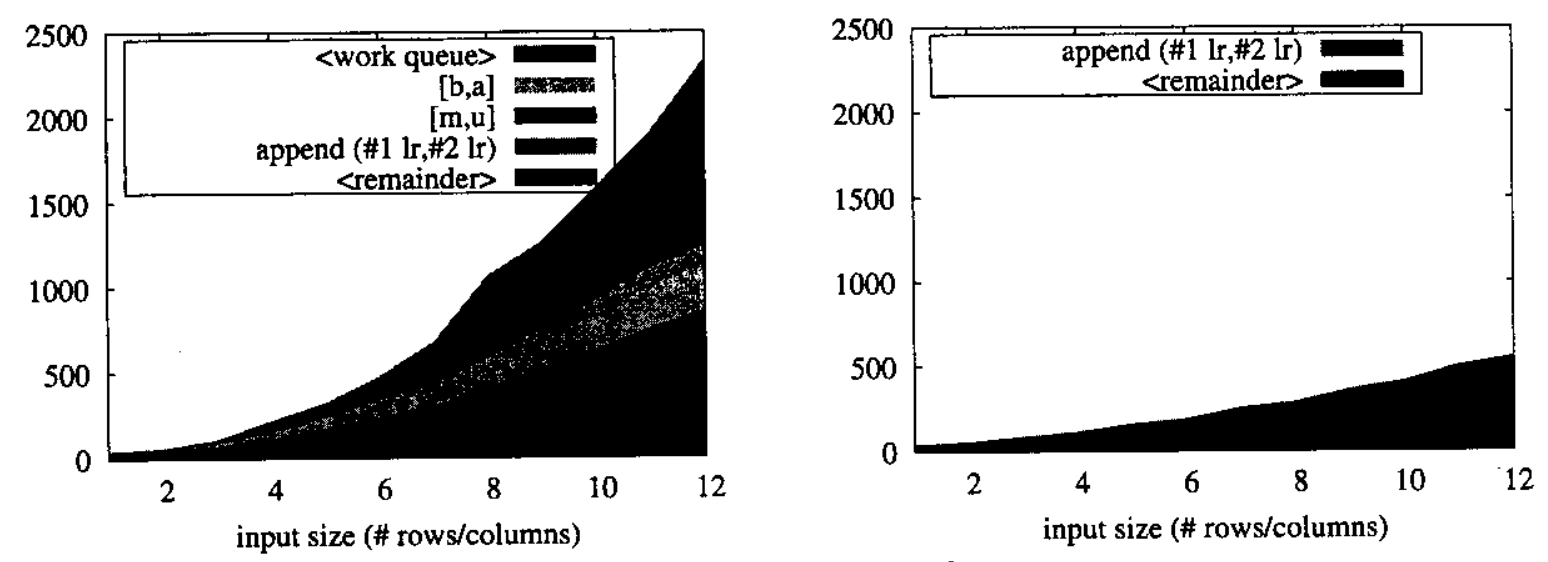

(b) space high-water mark 
Figure 3 Language Syntax. We use a call-by-value functional language with recursive functions, parallel pairs, and booleans. Components of pairs written $\left\{e_{1}, e_{2}\right\}$ may be computed in parallel. We include a separate class of values with annotations that capture sharing explicitly, but these values do not appear in the surface syntax used by programmers.

$\begin{array}{llll}\text { (expressions) } & e::= & x \mid \text { fun } f(x)=e\left|e_{1} e_{2}\right|\left\{e_{1}, e_{2}\right\} \mid \# i e \\ & & \text { true } \mid \text { false } \mid \text { if } e_{1} \text { then } e_{2} \text { else } e_{3} \mid v \\ \text { (values) } & v & ::=\langle f . x . e\rangle^{\ell} \mid\left\langle v_{1}, v_{2}\right\rangle^{\ell} \\ \text { (locations) } & \ell \in & L\end{array}$

These nodes represent the evaluation of the body of $v \mathrm{vm}$ and correspond to the top three entries in Figure 2(b).

We have presented a simple example here but the framework and tools also apply to complex programs with irregular parallel structure.

\section{Cost Semantics}

The cost semantics for our language is an evaluation semantics that computes both a result and an abstract cost reflecting how that result is obtained. It assigns a single cost to each closed program that enables us to construct a model of parallel execution and reason about the behavior of different scheduling policies.

In general, a cost semantics is necessary for any asymptotic analysis of running time or space use. For sequential implementations, there is an obvious cost semantics that nearly all programmers understand implicitly. For languages that fix the order of evaluation, the source code contains all the information necessary to reason about performance.

In this work, we give a cost semantics that serves as a basis for the asymptotic analysis of parallel programs, including their use of space. We believe that it is important that this semantics assigns costs to source-level programs. However, since the performance of programs depends on some aspects of the implementation, we must further interpret the results of the cost semantics, as discussed in Sections 3.2 and 3.3 below.

Figure 3 shows the fragment of our language we discuss in this section. In the implementation of our profiler, we extend this fragment with integers, floating-point numbers, lists, trees, and arrays, but none of these extensions prove difficult. We also include two forms of pair construction in our implementation: one that always evaluates components of the pair sequentially and one where components may be evaluated in parallel. Finally, we assume that all values are allocated in the heap for the sake of clarity, but this assumption may also be relaxed.

\subsection{Semantics}

A cost semantics is a dynamic semantics and thus yields results only for closed expressions, i.e. for a given program over a particular input. Just as in ordinary performance profiling, we must run a program over a series of inputs before we can generalize its behavior.

Our cost semantics is defined by the following judgment, which is read, expression e evaluates to value $v$ with computation graph $g$ and heap graph $h$.

$$
e \Downarrow v ; g ; h
$$

The extensional portions of this judgment are standard in the way that they relate expressions to values. As discussed below, edges in a computation graph represent control dependencies in the execution of a program, while edges in a heap graph represent dependencies on and between heap values. Rules for our cost semantics are shown in Figure 4.

As in the work of Blelloch and Greiner [1996], computation graphs $g$ are directed, acyclic graphs with exactly one source node (with in-degree 0 ) and one sink node (with out-degree 0 ) (i.e. directed series-parallel 
Figure 4 Profiling Cost Semantics. This semantics yields two graphs that can be used to reason about the parallel performance of programs. Computation graphs $g$ record dependencies in time while heap graphs $h$ record dependencies among values. Several omitted rules (e.g., E-IFFALSE) follow from their counterparts here. The locations of an expression $\operatorname{locs}(e)$ or a value $\operatorname{loc}(v)$ are the outermost locations of that expression or value. These auxiliary functions are defined in Appendix A.1.

$$
\begin{aligned}
& e \Downarrow v ; g ; h \\
& \frac{(\ell \text { fresh })}{\text { fun } f(x)=e \Downarrow\langle f . x . e\rangle^{\ell} ;[\ell] ;\left\{\left(\ell, \ell^{\prime}\right)\right\}_{\ell^{\prime} \in \operatorname{locs}(e)}}(\mathrm{E}-\mathrm{FUN}) \quad \frac{(n \text { fresh })}{v \Downarrow v ;[n] ; \emptyset}(\mathrm{E}-\mathrm{VAL}) \\
& \frac{e_{1} \Downarrow\left\langle f . x . e_{3}\right\rangle^{\ell_{1}} ; g_{1} ; h_{1} \quad e_{2} \Downarrow v_{2} ; g_{2} ; h_{2} \quad\left[\left\langle f . x . e_{3}\right\rangle^{\ell_{1}} / f\right]\left[v_{2} / x\right] e_{3} \Downarrow v_{3} ; g_{3} ; h_{3} \quad(n \text { fresh) }}{e_{1} e_{2} \Downarrow v_{3} ; g_{1} \oplus g_{2} \oplus[n] \oplus g_{3} ; h_{1} \cup h_{2} \cup h_{3} \cup\left\{\left(n, \ell_{1}\right),\left(n, \operatorname{loc}\left(v_{2}\right)\right)\right\}} \text { (E-APP) } \\
& \frac{e_{1} \Downarrow v_{1} ; g_{1} ; h_{1} \quad e_{2} \Downarrow v_{2} ; g_{2} ; h_{2} \quad(\ell \text { fresh })}{\left\{e_{1}, e_{2}\right\} \Downarrow\left\langle v_{1}, v_{2}\right\rangle^{\ell} ; g_{1} \otimes g_{2} \oplus[\ell] ; h_{1} \cup h_{2} \cup\left\{\left(\ell, \operatorname{loc}\left(v_{1}\right)\right),\left(\ell, \operatorname{loc}\left(v_{2}\right)\right)\right\}} \text { (E-FORK) } \\
& \frac{e \Downarrow\left\langle v_{1}, v_{2}\right\rangle^{\ell} ; g ; h \quad(n \text { fresh })}{\# i e \Downarrow v_{i} ; g \oplus[n] ; h \cup\{(n, \ell)\}}\left(\mathrm{E}-\mathrm{PROJ}_{i}\right) \quad \frac{(\ell \text { fresh })}{\text { true } \Downarrow \text { true }^{\ell} ;[\ell] ; \emptyset} \text { (E-TRUE) } \\
& e_{1} \Downarrow \text { true }^{\ell_{1}} ; g_{1} ; h_{1} \quad e_{2} \Downarrow v_{2} ; g_{2} ; h_{2} \quad(n \text { fresh }) \\
& \text { if } e_{1} \text { then } e_{2} \text { else } e_{3} \Downarrow v_{2} ; g_{1} \oplus\{n] \oplus g_{2} ; h_{1} \cup h_{2} \cup\left\{\left(n, \ell_{1}\right)\right\} \cup\{(n, \ell)\}_{\ell \in \operatorname{locs}\left(e_{3}\right)}
\end{aligned}
$$

graphs). Nodes are denoted $\ell$ and $n$ (and variants). Edges in the computation graph point forward in time. An edge from $n_{1}$ to $n_{2}$ indicates that $n_{1}$ must be executed before $n_{2}$. Each computation graph consists of a single-node, or of the sequential or parallel composition of smaller graphs. Graphs are written as tuples such as $\left(n_{s}, n_{e}, E\right)$ where $n_{s}$ is the source or start node, $n_{e}$ is the sink or end node, and $E$ is a set of edges. The remaining nodes of the graph are implicitly defined by the edge set. A graph consisting of a single node $n$ is written $(n, n, \emptyset)$ or simply $[n]$. Graph operations are defined below. Operands are shown first while the results are shown both graphically and symbolically below. (Here, a gray diamond stands for an arbitrary subgraph.)

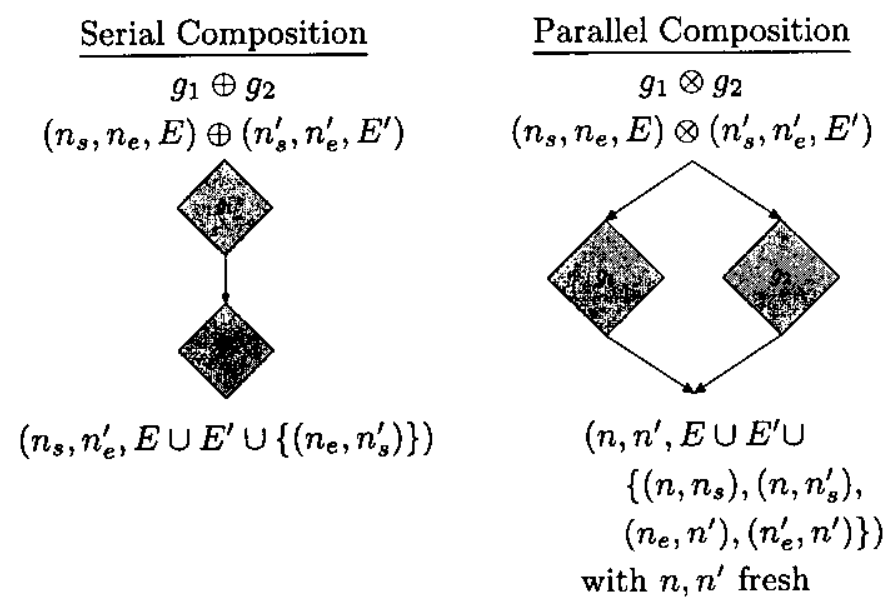

In the parallel composition on the right, the node $n$ is called the fork point and the node $n^{\prime}$ is called the join point.

We extend the work of Blelloch and Greiner [1996] with heap graphs. Heap graphs are also directed, acyclic graphs but do not have distinguished start or end nodes. Each heap graph shares nodes with the 
computation graph arising from the same execution. In a sense, computation and heap graphs may be considered as two sets of edges on a shared set of nodes. As above, the nodes of heap graphs are left implicit.

While edges in the computation graph point forward in time, edges in the heap graph point backward in time. If there is an edge from $n$ to $\ell$ then $n$ depends on the value at location $\ell$. It follows that any space associated with $\ell$ cannot be reclaimed until after $n$ has executed and any space associated with $n$ has also been reclaimed.

Edges in the heap graph record both the dependencies among heap values and dependencies on heap values by other parts of the program state. As an example of the first case, in the evaluation rule for parallel pairs (E-FORK), two edges are added to the heap graph to represent the dependencies of the pair on each of its components. Thus, if the pair is reachable, so is each component. In the evaluation of a function application (E-APP), however, two edges are added to express the use of heap values. The first such edge marks a use of the function. The second edge is more subtle and denotes a possible last use of the argument. For strict functions, this second edge is redundant: there will be another edge leading to the argument when it is used. However, for non-strict functions, this is the first point at which the garbage collector might reclaim the space associated with the argument. A similar issue is raised in the rules for conditionals. In E-IFTRUE, the semantics must record the locations that appear in the branch that is not taken. (In this case, these are the locations of $e_{3}$.) Again, the intuition is that this is the first point at which the storage corresponding to these locations might be reclaimed. In a sense, these edges represent our imperfect knowledge of program behavior at runtime: even though $e_{3}$ will never be executed, that fact is not known until the conditional is executed.

While there is some flexibility in designing these rules in our cost semantics, we choose the versions presented here because they can be implemented in a reasonable way and yet seem to constrain the implementation sufficiently. Care must be taken, however, because the implications of rules are sometimes subtle-see the example in Section 8.1.

\subsection{Schedules}

Together, the computation and heap graphs enable a programmer to analyze the behavior of her program under a variety of scheduling policies and numbers of processors. For a given program, each policy and processor count will give rise to a particular parallel execution that is determined by the constraints described by the computation graph $g$.

Definition (Schedule). A schedule of a graph $g=\left(n_{s}, n_{e}, E\right)$ is a sequence of sets of nodes $N_{0}, \ldots, N_{k}$ such that $n_{e} \in N_{k}$ and for all $i \in[0, k)$,

- $N_{i} \subseteq N_{i+1}$, and

- for all $n \in N_{i+1}$, $\operatorname{pred}_{g}(n) \subseteq N_{i}$.

Here, $\operatorname{pred}_{g}(n)$ is the set of nodes $n^{\prime}$ such that there is an edge $\left(n^{\prime}, n\right)$ in $g$. To restate, a schedule is a traversal of the computation graph where each node may only be visited after all of its parents and where several nodes may be visited in the same step. For expressions written by a programmer, we will generally be interested only in schedules where $N_{0}=\emptyset$. In some proofs the following section, however, we will need to consider expressions that have been partially evaluated and (therefore) schedules that start with a non-empty set.

\subsection{Roots}

To understand the use of space, the programmer must also account for the structure of the heap graph $h$. Given a schedule $N_{0}, \ldots, N_{k}$ for a graph $g$, consider the moment of time represented by some $N_{i}$. Because $N_{i}$ contains all previously executed nodes and because edges in $h$ point backward in time, each edge $(n, \ell)$ in $h$ will fall into one of the following three categories. 
- Both $n, \ell \notin N_{i}$. As the value associated with $\ell$ has not yet been allocated, the edge $(n, \ell)$ does not contribute to the use of space at time $i$.

- Both $n, \ell \in N_{i}$. While the value associated with $\ell$ has been allocated, the use of this value represented by this edge is also in the past. Again, the edge $(n, \ell)$ does not contribute to the use of space at time $i$.

- $\ell \in N_{i}$, but $n \notin N_{i}$. The value associated with $\ell$ has already been allocated, and $n$ represents a possible use in the future. Here, the edge $(n, \ell)$ does contribute to the use of space at time $i$.

In the definition below, we must also explicitly account for the location of the final value resulting from evaluation. Though this value may never be used in the program itself, we must include it when computing space use.

Definition (Roots). Given $e \Downarrow v ; g ; h$ and a schedule $N_{i}, i=0, \ldots, k$ of $g$, the roots after the evaluation of the nodes in $N_{i}$, written $\operatorname{roots}_{v, h}\left(N_{i}\right)$, is the set of nodes $\ell$ in $N_{i}$ where $\ell=\operatorname{loc}(v)$ or $h$ contains an edge leading from outside $N_{i}$ to $\ell$. Symbolically,

$$
\operatorname{roots}_{v, h}\left(N_{i}\right)=\left\{\ell \in N_{i} \mid \ell=\operatorname{loc}(v) \vee\left(\exists n .(n, \ell) \in h \wedge n \notin N_{i}\right)\right\}
$$

(The location of a value $\operatorname{loc}(v)$ is the outermost location of a value as defined in Appendix A.1.)

We use the term roots to evoke a related concept from work in garbage collection. For the reader who is most comfortable thinking in terms of an implementation, the roots might correspond to those memory locations that are reachable directly from the processor registers or the call stack. In the case of a parallel implementation, it also includes those locations that are reachable directly from the scheduler queue.

The definition of schedules given above includes any execution that respects the dependencies present in the original program. This includes schedules that use an unbounded amount of parallelism in a single step as well as those that stall execution for an unbounded length of time. We can refine this definition to limit ourselves to more realistic classes of schedulers and even to particular policies. One advantage of using cost graphs is that such refinements can be stated in a simple and clear manner. For example, a $p$-bounded scheduler uses at most $p$ processors at each step. In terms of the definition above,

$$
\forall i \text { such that } 0<i \leq k,\left|N_{i} \backslash N_{i-1}\right| \leq p
$$

Similarly, the behavior of a depth-first scheduler should correspond to a depth-first traversal of the computation graph.

\section{Provable Implementations}

While the evaluation semantics given in the previous section allows a programmer to draw conclusions about the performance of her program, these conclusions would be meaningless if the implementation of the language did not reflect the costs given by that semantics. In this section, we define several provable implementations [Blelloch and Greiner, 1996] of this language, each as a transition (small-step) semantics. The first "implementation" is a non-deterministic semantics that defines all possible parallel executions. Each subsequent semantics will define the behavior of a particular scheduling algorithm. The following table gives a brief overview of all the semantics used in this report.

\begin{tabular}{|l|l|c|l|}
\hline Semantics (Figure) & Style & Judgment(s) & Notes \\
\hline \hline cost (4) & big-step & $e \Downarrow v ; g ; h$ & sequential, profiling semantics \\
\hline primitive (5) & small-step & $e \longrightarrow e^{\prime}$ & $\begin{array}{l}\text { axioms shared among parallel imple- } \\
\text { mentations }\end{array}$ \\
\hline non-deterministic (6) & small-step & $\begin{array}{l}e \stackrel{\text { nd }}{\longrightarrow} e^{\prime} \\
\stackrel{\text { nd }}{\rightleftarrows} d^{\prime}\end{array}$ & defines all possible parallel executions \\
\hline depth-first (7) & small-step & $\begin{array}{l}e \stackrel{\text { df }}{\rightleftarrows} e^{\prime} \\
d \stackrel{\text { df }}{\longrightarrow} d^{\prime}\end{array}$ & $\begin{array}{l}\text { algorithmic implementation favoring } \\
\text { left-most sub-expressions }\end{array}$ \\
\hline
\end{tabular}


Figure 5 Primitive Transitions. These rules encode transitions where no parallel execution is possible. They will be used in each of the different scheduling semantics that follow in this section. The substitution of a value declaration into an expression $[\delta] e$ is defined in Appendix A.2.

$$
\begin{aligned}
& e \longrightarrow e^{\prime} \\
& \frac{(\ell \text { fresh })}{\text { fun } f(x)=e \longrightarrow\langle f \cdot x \cdot e\rangle^{\ell}} \text { (P-FUN) }
\end{aligned}
$$

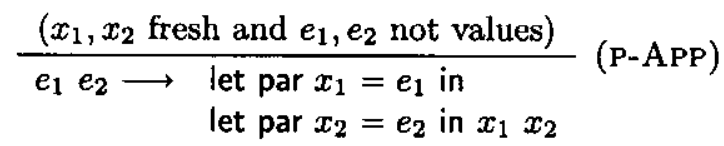

$$
\begin{aligned}
& \overline{\langle f . x . e\rangle^{\ell} v_{2} \longrightarrow\left[\langle f . x . e\rangle^{\ell} / f\right]\left[v_{2} / x\right] e} \text { (P-APPBETA) } \\
& \frac{(\ell \text { fresh })}{\left\{v_{1}, v_{2}\right\} \longrightarrow\left\langle v_{1}, v_{2}\right\rangle^{\ell}} \text { (P-PAIR) } \quad \frac{(x \text { fresh and } e \text { not a value })}{\# i e \longrightarrow \text { let par } x=e \text { in } \# i x}\left(\text { P-PROJ }_{i}\right)
\end{aligned}
$$

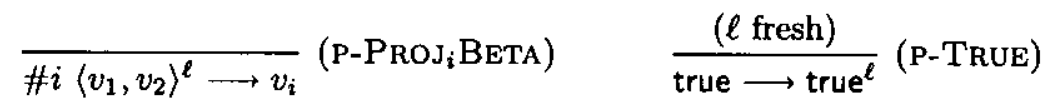

$$
\begin{aligned}
& \frac{\left(x \text { fresh and } e_{1} \text { not a value }\right)}{\text { if } e_{1} \text { then } e_{2} \text { else } e_{3} \longrightarrow \text { let par } x=e_{1} \text { in if } x \text { then } e_{2} \text { else } e_{3}} \text { (P-IF) } \\
& \text { if true }{ }^{\ell} \text { then } e_{2} \text { else } e_{3} \longrightarrow e_{2} \text { (P-IFTRUE) } \\
& \frac{\left(x_{1}, x_{2} \text { fresh and } e_{1}, e_{2} \text { not values }\right)}{\left\{e_{1}, e_{2}\right\} \longrightarrow \quad \text { let par } x_{1}=e_{1}} \text { (P-FoRK) } \\
& \overline{\text { let } \operatorname{par} \delta \text { in } e \longrightarrow[\delta] e} \text { (P-JoIN) }
\end{aligned}
$$

As part of the implementation of this language, we extend the syntax to include a parallel let construct. This construct is used to denote expressions whose parallel evaluation has begun but not yet finished. Declarations within a let par may step in parallel, depending on the constraints enforced by one of the transition semantics below. Declarations and let par expressions reify a stack of expression contexts such as those that appear in many abstract machines (e.g. [Landin, 1964]). Unlike a stack, which has exactly one topmost element, there are many "leaves" in our syntax that may evaluate in parallel. These extensions are shown below.

$$
\begin{array}{llll}
\text { (expressions) } & e & :=\ldots \mid \text { let } \operatorname{par} d \text { in } e \\
\text { (declarations) } & d & :=x=e \mid d_{1} \text { and } d_{2} \\
\text { (value declarations) } & \delta & :=x=v \mid \delta_{1} \text { and } \delta_{2}
\end{array}
$$

To facilitate the definition of several different parallel semantics, we first factor out those parts of the semantics that are common to each variation. These primitive sequential transitions are defined by the following judgment.

$$
e \longrightarrow e^{\prime}
$$

This judgment represents the step taken by a single processor in one unit of time (e.g., allocating a pair, applying a function). Primitive transitions are defined by the axioms in Figure 5. These axioms limit where parallel evaluation may occur by defining the intermediate forms for the evaluation of pairs and function application. When exactly parallel evaluation occurs is defined by the scheduling semantics, as given in the remainder of this section. 
Figure 6 Non-Deterministic Parallel Transition Semantics. This semantics defines all possible parallel transitions of an expression, including those that take an arbitrary number of primitive steps in parallel. Parallelism is isolated within transition expressions of the form let par. Declarations step in parallel using ND-BRANCH. Note that expressions (or portions thereof) may remain unchanged using the rule ND-IDLE.

$\stackrel{\text { nd }}{\longmapsto} e^{\prime}$

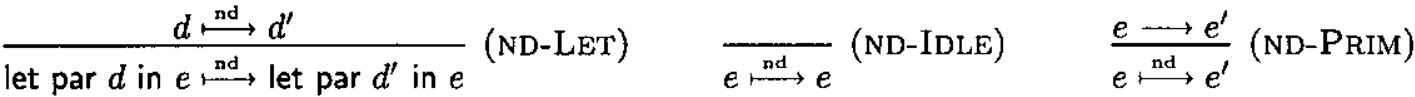

$d \stackrel{\text { nd }}{\longmapsto} d^{\prime}$

$$
\frac{e \stackrel{\text { nd }}{\longmapsto} e^{\prime}}{x=e \stackrel{\text { nd }}{\longmapsto} x=e^{\prime}} \text { (ND-LEAF) } \quad \frac{d_{1} \stackrel{\text { nd }}{\longmapsto} d_{1}^{\prime} \quad d_{2} \stackrel{\text { nd }}{\longmapsto} d_{2}^{\prime}}{d_{1} \text { and } d_{2} \stackrel{\text { nd }}{\longmapsto} d_{1}^{\prime} \text { and } d_{2}^{\prime}} \text { (ND-BRANCH) }
$$

\subsection{Non-Deterministic Scheduling}

The first implementation in this report is a non-deterministic ND transition semantics that defines all possible parallel executions. Though this semantics itself does not serve as a model for a realistic implementation, it is a useful tool in reasoning about other, more realistic, semantics. The non-deterministic semantics is defined by a pair of judgments

$$
e \stackrel{\text { nd }}{\longmapsto} e^{\prime} \quad d \stackrel{\text { nd }}{\longmapsto} d^{\prime}
$$

that state, expression $e$ takes a single parallel step to $e^{\prime}$ and, similarly, declaration $d$ takes a single parallel step to $d^{\prime}$. This semantics allows unbounded parallelism: it models execution on a parallel machine with an unbounded number of processors. It is defined by the rules in Figure 6.

Most of the ND rules are straightforward. The only non-determinism lies in the application of the rule ND-IDLE. In a sense, this rule is complemented by ND-BRANCH: The latter says that all branches may be executed in parallel, but the former allows any sub-expression to sit idle during a given parallel step.

\subsubsection{Extensional Behavior}

Though this implementation is non-deterministic in how it schedules parallel evaluation, the result of ND evaluation will always be the same, no matter which expressions evaluate in parallel. This statement is formalized in the following theorem. (In this and other results below, we always consider equality up to the renaming of locations.)

Theorem 1 (Confluence). If $e \stackrel{\text { nd }}{\longmapsto} e^{\prime}$ and $e \stackrel{\text { nd }}{\longmapsto} e^{\prime \prime}$ then there exists an expression $e^{\prime \prime \prime}$ such that $e^{\prime} \stackrel{\text { nd }}{\longmapsto}^{*} e^{\prime \prime \prime}$ and $e^{\prime \prime} \stackrel{n d}{\longmapsto}^{*} e^{\prime \prime \prime}$. Similarly, $d \stackrel{\text { nd }}{\longmapsto}{ }^{*} d^{\prime}$ and $d \stackrel{\text { nd }}{\longmapsto} * d^{\prime \prime}$ then there exists a declaration $d^{\prime \prime \prime}$ such that $d^{\prime} \longmapsto^{n d}{ }^{*} d^{\prime \prime \prime}$ and $d^{\prime \prime} \stackrel{\text { nd }}{\longmapsto} d^{\prime \prime \prime}$.

Following Huet [1980], we can use strong confluence (Lemma 1) to conclude that $\stackrel{\text { nd }}{\longmapsto}$ is confluent. An explicit proof appears in Appendix B.1. We note because the ND semantics allows infinite sequences of transitions (even for expressions where the cost semantics gives a finite derivation), local confluence is not sufficient to prove confluence.

Lemma 1 (Strong Confluence). If $e \stackrel{\text { nd }}{\longmapsto} e^{\prime}$ and $e \stackrel{\text { nd }}{\longmapsto} e^{\prime \prime}$ then there exists an expression $e^{\prime \prime \prime}$ such that $e^{\prime} \stackrel{\text { nd }}{\longmapsto}^{*} e^{\prime \prime \prime}$ and $e^{\prime \prime} \stackrel{\text { nd }}{\longmapsto} e^{\prime \prime \prime}$. Similarly, $d \stackrel{\text { nd }}{\longmapsto} d^{\prime}$ and $d \stackrel{\text { nd }}{\longmapsto} d^{\prime \prime}$ then there exists a declaration $d^{\prime \prime \prime}$ such that $d^{\prime} \stackrel{\text { nd }}{\longmapsto} * d^{\prime \prime \prime}$ and $d^{\prime \prime} \stackrel{\text { nd }}{\longmapsto} d^{\prime \prime \prime}$. 
The lemma statement is also shown in the following illustration. Given the solid arrows, we would like to show the existence of $e^{\prime \prime \prime}, d^{\prime \prime \prime}$, and the dotted arrows.
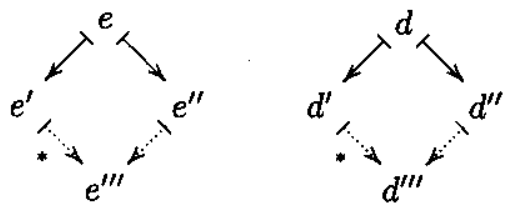

Proof. By simultaneous induction on the derivations of $e \stackrel{\text { nd }}{\longmapsto} e^{\prime}$ and $d \stackrel{\text { nd }}{\longmapsto} d^{\prime}$.

Case ND-IDLE: In this case $e^{\prime}=e$. Assume that $e \stackrel{\text { nd }}{\longmapsto} e^{\prime \prime}$ was derived using rule $R$. Let $e^{\prime \prime \prime}=e^{\prime \prime}$. Then we have $e \stackrel{\text { nd }}{\longmapsto} e^{\prime \prime}$ (by applying $R$ ) and $e^{\prime \prime} \stackrel{\text { nd }}{\longmapsto} e^{\prime \prime}$ (by ND-IDLE), as required.

As all of the non-determinism in this semantics is focused in the use of the ND-IDLE rule, the remaining cases follow from an application of the induction hypothesis and the given rule. One example is shown here. Case ND-LET: In this case $e=$ let par $d$ in $\hat{e}$ and the rule applied on the left side is as follows.

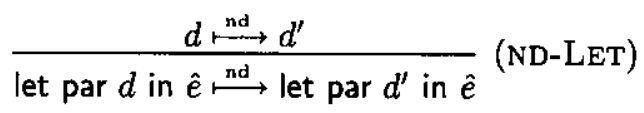

One of three rules was applied on the right side.

Subcase ND-IDLE: Then $e^{\prime \prime}=e$. Let $e^{\prime \prime \prime}=e^{\prime}$. We have $e^{\prime \prime} \stackrel{\text { nd }}{\longrightarrow} e^{\prime \prime \prime}$ by rule ND-LET and the derivation above; $e^{\prime} \stackrel{\text { nd }}{\longmapsto} e^{\prime \prime \prime}$ by ND-IDLE as required.

Subcase ND-LET: Then $e^{\prime \prime}=$ let par $d^{\prime \prime}$ in $\hat{e}$. By the induction hypothesis, there exists a declaration $d^{\prime \prime \prime}$ such that $d^{\prime} \stackrel{\text { nd }}{\longmapsto} d^{\prime \prime \prime}$ and $d^{\prime \prime} \stackrel{\text { nd }}{\longmapsto} d^{\prime \prime \prime}$. For each transition starting from $d^{\prime}$ and $d^{\prime \prime}$, we apply ND-LET to derive the following transitions.

$$
\begin{aligned}
& \text { let par } d^{\prime} \text { in } \hat{e} \stackrel{\text { nd }}{\longmapsto} \text { * let par } d^{\prime \prime \prime} \text { in } \hat{e} \\
& \text { let par } d^{\prime \prime} \text { in } \hat{e} \stackrel{\text { nd }}{\longmapsto} \text { let par } d^{\prime \prime \prime} \text { in } \hat{e}
\end{aligned}
$$

Subcase ND-PRIM: From the form of $e$, the only primitive transition that applies is P-JoIN. Thus $d$ is a value declaration. It follows that $d^{\prime}=d$ and therefore, $e^{\prime}=e$. Let $e^{\prime \prime \prime}=e^{\prime \prime}$. We have $e^{\prime} \stackrel{\text { nd }}{\longmapsto} e^{\prime \prime \prime}$ by ND-PRIM and ND-JOIN; $e^{\prime \prime} \stackrel{\text { nd }}{\longmapsto} e^{\prime \prime \prime}$ by ND-IDLE.

Before considering the intensional behavior of the parallel semantics, we prove several properties relating it extensional behavior to that of the cost semantics. As such, we temporarily ignore the cost graphs and write $e \Downarrow v$ if $e \Downarrow v ; g ; h$ for some $g$ and $h$. The first such property (Completeness) states that any result obtained using the cost semantics can also be obtained using the ND implementation.

Theorem 2 (ND Completeness). If $e \Downarrow v$ then $e^{\stackrel{n d}{\longleftrightarrow}} * v$.

The proof is carried out by induction on the derivation of $e \Downarrow v$ and is shown in Appendix B.2.

The following theorem (Soundness) ensures that any result obtained by the implementation semantics can also be derived using the cost semantics. As the extensions to the source language given in this section represent runtime intermediate forms, we define an embedding of these new expression forms into the original syntax. Parallel let expressions are embedded by substituting away bound variables. Declarations are flattened by the embedding.

$$
\begin{array}{ll}
\text { rlet par } d \text { in } e\urcorner & =\lceil\ulcorner d\urcorner]\ulcorner e\urcorner \\
\ulcorner e\urcorner & =e \quad(e \neq \text { let par } d \text { in } e) \\
\left\ulcorner x=e^{\urcorner}\right\urcorner & =x=\ulcorner e\urcorner \\
\left\ulcorner d_{1} \text { and } d_{2}\right\urcorner & =\left\ulcorner d_{1}\right\urcorner \text { and }\left\ulcorner d_{2}\right\urcorner
\end{array}
$$


We also define a vectorized form of the evaluation relation that evaluates declarations in parallel.

$$
\frac{e \Downarrow v}{x=e \Downarrow x=v} \quad \frac{d_{1} \Downarrow \delta_{1} \quad d_{2} \Downarrow \delta_{2}}{d_{1} \text { and } d_{2} \Downarrow \delta_{1} \text { and } \delta_{2}}
$$

Theorem 3 (ND Soundness). If $e \stackrel{\text { nd }}{\longrightarrow} v$, then $\ulcorner e\urcorner \Downarrow v$. Similarly, if $d \stackrel{\text { nd }}{\longrightarrow} \delta$, then $\ulcorner d\urcorner \Downarrow \delta$.

Proof. By induction on the number of steps $n$ in the sequence of transitions.

Case 0: In this case, $e=v$. Since every value is related to itself in the cost semantics, the case is proved. The same applies to $d$ and $\delta$.

Case $n>0$ : Here we have, $e \stackrel{\text { nd }}{\longmapsto} e^{\prime}$ and $e^{\prime} \stackrel{\text { nd }}{\longmapsto} * v$. Inductively, we have $\left\ulcorner e^{\prime}\right\urcorner \Downarrow v$. The remainder of this case is given by the following lemma.

Lemma 2. If $e \stackrel{\text { nd }}{\longmapsto} e^{\prime}, e^{\prime} \stackrel{n d}{\longmapsto} v$, and $\left\ulcorner e^{\prime}\right\urcorner \Downarrow v$, then $\left\ulcorner e^{\urcorner} \Downarrow v\right.$. Similarly, if $d^{\stackrel{n d}{\longmapsto}} d^{\prime}, d^{\prime} \stackrel{n d}{\longmapsto} \stackrel{n}{*}^{*}$ and $\left\ulcorner d^{\prime}\right\urcorner \Downarrow \delta$, then $\ulcorner d\urcorner \Downarrow \delta$.

The proof is carried out by induction on the derivations of $e \stackrel{\text { nd }}{\longmapsto} e^{\prime}$ and $d \stackrel{\text { nd }}{\longmapsto} d^{\prime}$ and is given in Appendix B.3.

\subsubsection{Intensional Behavior}

Having considered the extensional behavior of this implementation, we now turn to its intensional behavior. As we take the semantics to define all possible parallel executions, it should be the case the any schedule we derive from the cost semantics is implemented by a sequence of parallel steps, as defined by the transition relation. This statement is made precise in the following theorem.

Theorem 4 (Cost Completeness). If $e \Downarrow v ; g ; h$ and $N_{0}, \ldots, N_{k}$ is a schedule of $g$ with $N_{0}=\operatorname{locs}(e)$ then there exists a sequence of expressions $e_{0}, \ldots, e_{k}$ with $e_{0}=e$ and $e_{k}=v$ and for all $i \in[0, k), e_{i} \stackrel{n d}{\longrightarrow} e_{i+1}$ and $\operatorname{locs}\left(e_{i}\right) \subseteq \operatorname{roots}_{v, h}\left(N_{i}\right)$.

The final condition of the theorem states that the use of space in the parallel semantics, as determined by $\operatorname{locs}()$, is approximated by the measure of space in the cost graphs, as given by roots(). This theorem is proved much like its extensional counterpart above, by induction on the derivation of $e \Downarrow v ; g ; h$. The freshness conditions in the cost semantics are critical in this proof. They allow us to reason inductively about each sub-expression and to divide and recombine the schedules corresponding to these sub-expressions.

\subsection{Depth-First Scheduling}

We now define an alternative transition semantics that is deterministic and implements a parallel depthfirst schedule. Depth-first (DF) schedules [Blelloch et al., 1999], defined below, prioritize the leftmost subexpressions of a program and always complete the evaluation of these leftmost sub-expressions before proceeding to sub-expressions on the right. The semantics in this section implements a $p$-depth-first $(p$-DF) scheduler, a scheduler that uses at most $p$ processors. As a trivial example, a left-to-right sequential evaluation is equivalent to a one processor or 1-DF schedule.

Just as we defined the non-deterministic implementation as a transition relation, we can do the same for the depth-first implementation. The $p$-DF transition semantics is defined on configurations $p ; e$ and $p ; d$. These configurations describe an expression or declaration together with an integer $p$ that indicates the number of processors that have not yet been assigned a task in this parallel step. At the root of the derivation of each parallel step, $p$ will be equal to the total number of processors. Within a derivation, $p$ may be smaller but not less than zero. The semantics is given by the following pair of judgments.

$$
p ; e \stackrel{\text { df }}{\longmapsto} p^{\prime} ; e^{\prime} \quad p ; d \stackrel{\text { df }}{\longmapsto} p^{\prime} ; d^{\prime}
$$

These judgments define a single parallel step of an expression or declaration. The first is read, given $p$ available processors, expression e steps to expression $e^{\prime}$ with $p^{\prime}$ processors remaining unused. The second has an analogous meaning for declarations. 
Figure $7 p$-Depth-First Parallel Transition Semantics. This deterministic semantics defines a single parallel step for left-to-right depth-first schedule using at most $p$ processors. Configurations $p ; e$ and $p ; d$ describe expressions and declarations with $p$ unused processors remaining in this time step.

$$
\begin{aligned}
& p ; e \stackrel{\text { df }}{\longmapsto} p^{\prime} ; e^{\prime} \\
& \frac{p ; d \stackrel{\text { df }}{\longmapsto} p^{\prime} ; d^{\prime}}{p \text {; let par } d \text { in } e \stackrel{\text { df }}{\longmapsto} p^{\prime} \text {; let par } d^{\prime} \text { in } e} \text { (DF-LET) } \quad \frac{}{p ; v \stackrel{\text { df }}{\longmapsto} p ; v} \text { (DF-VAL) }
\end{aligned}
$$

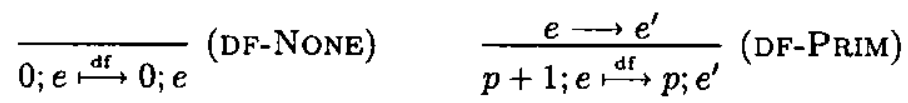

$$
\begin{aligned}
& p ; d \stackrel{\text { df }}{\longmapsto} p^{\prime} ; d^{\prime} \\
& \frac{p ; e \stackrel{\mathrm{d} f}{\longmapsto} p^{\prime} ; e^{\prime}}{p ; x=e \stackrel{\mathrm{d} f}{\longmapsto} p^{\prime} ; x=e^{\prime}} \text { (DF-LEAF) } \quad \frac{p ; d_{1} \stackrel{\mathrm{d} f}{\longmapsto} p^{\prime} ; d_{1}^{\prime} \quad p^{\prime} ; d_{2} \stackrel{\mathrm{df}}{\longmapsto} p^{\prime \prime} ; d_{2}^{\prime}}{p ; d_{1} \text { and } d_{2} \stackrel{\mathrm{d} f}{\longmapsto} p^{\prime \prime} ; d_{1}^{\prime} \text { and } d_{2}^{\prime}} \text { (DF-BRANCH) }
\end{aligned}
$$

The $p$-DF transition semantics is defined by the rules given in Figure 7. Most notable is the DF-BraNCH rule. It states that a parallel declaration may take a parallel step if any available processors are used first on the left sub-declaration and then any remaining available processors are used on the right. Like the non-deterministic semantics above, the $p$-DF transition semantics relies on the primitive transitions given in Figure 5. In rule DF-PRIM, one processor is consumed when a primitive transition is applied.

For the DF semantics, we must reset the number of available processors after each parallel step. To do so, we define a "top-level" transition judgment for DF evaluation with $p$ processors. This judgment is defined by exactly one rule, shown below. Note that the number of processors remaining idle $p^{\prime}$ remains unconstrained.

$$
\frac{p ; e \stackrel{\mathrm{df}}{\longmapsto} p^{\prime} ; e^{\prime}}{e \stackrel{\mathrm{p} \text {-df }}{\longmapsto} e^{\prime}}
$$

The complete evaluation of an expression, as for the non-deterministic semantics, is given by the reflexive, transitive closure of the transition relation $\stackrel{p=-d f}{\longrightarrow} *$

We now consider several properties of the DF semantics. First, unlike the non-deterministic implementation, this semantics defines a particular evaluation strategy.

Theorem 5 (Determinacy of DF Evaluation). If $p ; e \stackrel{d f}{\longmapsto} p^{\prime} ; e^{\prime}$ and $p ; e \stackrel{d J}{\longmapsto} p^{\prime \prime} ; e^{\prime \prime}$ then $p^{\prime}=p^{\prime \prime}$ and $e^{\prime}=e^{\prime \prime}$. Similarly, if $p ; d \stackrel{d f}{\longrightarrow} p^{\prime} ; d^{\prime}$ and $p ; d \stackrel{d y}{\longrightarrow} p^{\prime \prime} ; d^{\prime \prime}$ then $p^{\prime}=p^{\prime \prime}$ and $d^{\prime}=d^{\prime \prime}$.

The proof is carried out by induction on the first derivation and hinges on the following two facts: first, that DF-VAL and DF-VAL yield the same results, and second, that in no instance can both DF-LET and DF-PRIM be applied.

We can easily show the DF semantics is correct with respect to the cost semantics, simply by showing that its behavior is contained within that of the non-deterministic semantics.

Theorem 6 (DF Soundness). If $p ; e \stackrel{d f}{\longmapsto} p^{\prime} ; e^{\prime}$ then $e \stackrel{\text { nd }}{\longmapsto} e^{\prime}$. Similarly, If $p ; d \stackrel{d f}{\longmapsto} p^{\prime} ; d^{\prime}$ then $d \stackrel{\text { nd }}{\longmapsto} d^{\prime}$.

Proof. By induction on the derivation of $p ; e \stackrel{\text { di }}{\longmapsto} p^{\prime} ; e^{\prime}$. Cases for derivations ending with rules DF-LET, DF-LEAF, and DF-BRANCH follow immediately from appeals to the induction hypothesis and analogous rules in the non-deterministic semantics. DF-PRIM also follows from its analogue. Rules DF-NONE and DF-VAL are both restrictions of ND-IDLE. 
It follows immediately that if $e \stackrel{\text { p-df }}{\longmapsto} e^{\prime}$ then $e \stackrel{\text { nd }}{\longmapsto} e^{\prime}$. This result shows the benefit of defining and reasoning about a non-deterministic semantics: once we have shown the soundness of an implementation with respect to the non-deterministic semantics, we get soundness with respect to the cost semantics for free. Thus, we know there is some schedule that accurately models behavior of the DF implementation. It only remains to pick out precisely which schedule does so.

To allow programmers to understand the behavior of this semantics, we define a more restricted form of schedule. We must also refine the definition of graphs to allow an ordering of nodes. For each such computation graph $g$ there is a unique $p$-DF schedule. As shown below, these schedules precisely capture the behavior of the DF implementation.

Definition ( $p$-Depth-First Schedule). A $p$-depth-first schedule of a graph $g$ is a schedule $N_{0}, \ldots, N_{k}$ such that all $i \in[0, k)$,

- $\left|E_{i+1}\right| \leq p$ and

- for all $n \in N_{i+1}, n^{\prime} \in N_{i}, n^{\prime} \preceq n$,

and for any other schedule of $g$ given by $N_{0}^{\prime}, \ldots, N_{j}^{\prime}$ that meets these constraints, $n \in N_{i}^{\prime} \Rightarrow n \in N_{i}$.

Here $n^{\prime} \preceq n$ if $n^{\prime}$ appears first in $g$. The final condition ensures that the depth-first schedule is the most aggressive schedule that respects this ordering of nodes: if it is possible to execute node $n$ at time $i$ (as evidenced by its membership in $N_{i}^{\prime}$ ) then any depth-first schedule must also do so.

Theorem 7 (DF Cost Completeness). If $e \Downarrow v ; g ; h$ and $N_{0}, \ldots, N_{k}$ is a $p$-DF schedule of $g$ with $N_{0}=\operatorname{locs}(e)$ then there exists a sequence of expressions $e_{0}, \ldots, e_{k}$ with $e_{0}=e$ and $e_{k}=v$ and for all $i \in[0, k), e_{i} \stackrel{p \text { pdf }}{\longrightarrow}{ }^{*} e_{i+1}$ and $\operatorname{locs}\left(e_{i}\right) \subseteq \operatorname{roots}_{v, h}\left(N_{i}\right)$.

This theorem must be generalized over DF schedules which may use a different number of processors at each time step. This allows a $p$-DF schedule to be split into two DF schedules that, when run in parallel, never use more than $p$ processors in a given step. The proof relies on the fact that any DF schedule can be split in this fashion, and moreover, that it can be split so that the left-hand side is allocated all the processors (up to $p$ ) of which it could possibly make use.

\subsection{Breadth-First Scheduling}

Just as the semantics in the previous section captured the behavior corresponding to a parallel depth-first traversal of the computation graph, we can also give an implementation corresponding to a breadth-first (BF) traversal. This is the most "fair" schedule in the sense that it distributes computational resources evenly across parallel tasks. For example, given four parallel tasks, a 1-BF scheduler alternates round-robin between the four. A 2-BF scheduler takes one step for each of the first two tasks in parallel, followed by one step for the second two, followed again by the first two, and so on.

We omit the presentation of this semantics and only state that a theorem making a precise correspondence between breadth-first schedules and this implementation, similar to that shown above for the depth-first case, can also be proved.

\section{Profiling}

In addition to serving as a guide for the implementations in the previous section, our cost semantics also forms a basis for a suite of programmer tools. We have implemented our cost semantics as an interpreter, and the resulting cost graphs are used by the two tools described below. 


\subsection{Parallel Simulation}

Our simulator can be instantiated to measure many different performance characteristics, but each instance can be broken down into three parts: a generic component that maintains information about the graph traversal, an implementation of a scheduling policy, and a function that measures some aspect of program performance.

The simulator is a sequential program so implementing scheduling policies in the simulator requires no synchronization or other concurrency control. The breadth- and depth-first scheduling policies discussed above can each be implemented with a single line of SML using standard list primitives.

We focus on the high-water mark of memory use as the primary measure of performance. As described above, the roots of the heap graph at an intermediate point during execution represent those values that are immediately reachable by the program itself. The total space in use at one point during execution is determined by the set of all nodes in the heap graph reachable from these roots. We use the size of the set of reachable nodes in the heap graph as the measure of space use.

By iterating over different inputs, we can compute the high-water mark as a function of the input size. This allows us to plot results such as those shown in Figure 2(b).

\subsection{Visualization}

The cost graphs given by our semantics are often quite large and difficult to process visually. We have implemented a method that distills these graphs and yields their essential components. As the goal is to produce a meaningful visual output, we associate a size and color with each node and edge. (In this write-up, we restrict ourselves to black and shades of gray.) As the computation and heap graphs share nodes, we show one superimposed over the other. The resulting graph can then be rendered into various image formats by a freely available graph layout package. ${ }^{1}$ All of the graphs shown in this paper are mechanically generated from our cost semantics and an implementation of the following numbered rules. We determined these rules in part through experimentation, but in a large part upon the principles discussed below.

We are most interested in the parallel structure of program execution. Thus a series of nodes that describes sequential computation can be rendered as a single node. We use node size to indicate the amount of sequential computation and node position to indicate computational dependencies.

1. For each node $n$ in the computation graph if 1) $n$ has out-degree one, 2) $n$ has in-degree one, and 3) the (sole) predecessor of $n$ also has out-degree one, then coalesce $n$ with its predecessor. The area of the resulting node is the sum of the area of the two coalesced nodes. Nodes in the original graph are assigned unit area.

2. Remove edges between coalesced nodes in both the computation and heap graphs. (There are no self-edges in the result.)

3. Constrain the layout so that the vertical position of nodes respects the partial order determined by computation edges.

In the output graph, we draw computation edges in a light gray as the structure of the computation graph can be roughly derived from the layout of the nodes: among vertically aligned nodes, those closer to the top of the graph must be executed first. We also omit arrowheads on edges as they add to visual clutter. An example of node coalescing is shown here.

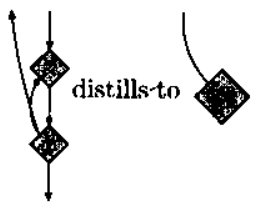

\footnotetext{
${ }^{1}$ Graphviz - Graph Visualization Software, http://www.graphviz.org/
} 
Due to the structure of the cost graphs, coalescing will never create non-unique edges in the computation graph (i.e., more than one edge between the same pair of nodes). On the other hand, it will often be the case that there are several heap edges between the same pair of nodes. We considered trying to represent these duplicate heap edges, for example, by weighting heap edges in the output according to number of duplicates. This, however, ignores any sharing among these heap edges and may lead to confusing visual results (e.g., a group of heavy edges that represents a relatively small amount of heap space): For graphs distilled independently of a particular point in time, duplicate heap edges are removed, and all heap edges are given the same weight.

If we restrict ourselves to a single moment in time, we can overcome the difficulties with representing sharing and also focus on the behavior of a specific scheduling policy and its effect on memory use. We use the color of both nodes and heap edges to highlight the behavior of the scheduling policy at time $i$, the moment when memory use reaches it high-water mark.

4. Fill nodes with black if they are executed at or before time $i$, and gray otherwise.

5. Draw heap edges that determine roots at time $i$ in black and other heap edges in gray.

When coloring nodes and edges according to these rules, we must be careful about which nodes we coalesce, as we expect those heap edges that determine roots to connect only executed and unexecuted nodes.

6. Avoid coalescing two nodes if one has been executed at or before time $i$ and the other has not.

Finally, now that we have restricted ourselves to a single moment in time, we can properly account for sharing in the heap graph.

7. Weight each heap edge according to its share of the total amount of heap space reachable from that edge at time $i$.

Thus the total space use at the high-water mark may be determined from the total weight of the black heap edges (i.e., those representing the roots). Generally, the above rules mean that the visual properties of distilled graphs can be interpreted according to the following table.

\begin{tabular}{l|l} 
The greater the $\ldots$ & then the more... \\
\hline graph height & sequential dependencies \\
graph width & possible parallelism \\
node size & computation \\
edge thickness & space use
\end{tabular}

\section{Implementation in MLton}

In this section we describe an implementation of a parallel functional language based on our semantics. This serves to validate our profiling results and demonstrate that implementations of our specification can achieve good parallel speed-ups.

Our implementation is an extension of MLton [Weeks, 2006], a whole-program, optimizing compiler for Standard ML [Milner et al., 1997]. This is the first parallel implementation of MLton. In keeping with the philosophy that performance-critical code can be written in a high-level language, we implemented as much as the runtime support for parallelism in SML as we could. That said, we were also required to make some changes and additions to the existing runtime system, which is written in $\mathrm{C}$.

\subsection{Runtime System}

MLton is comprised of a compiler, a set of libraries, and a uniprocessor runtime system. Our first task was to make modifications to the runtime to ensure that shared resources would be safely accessed by concurrently 


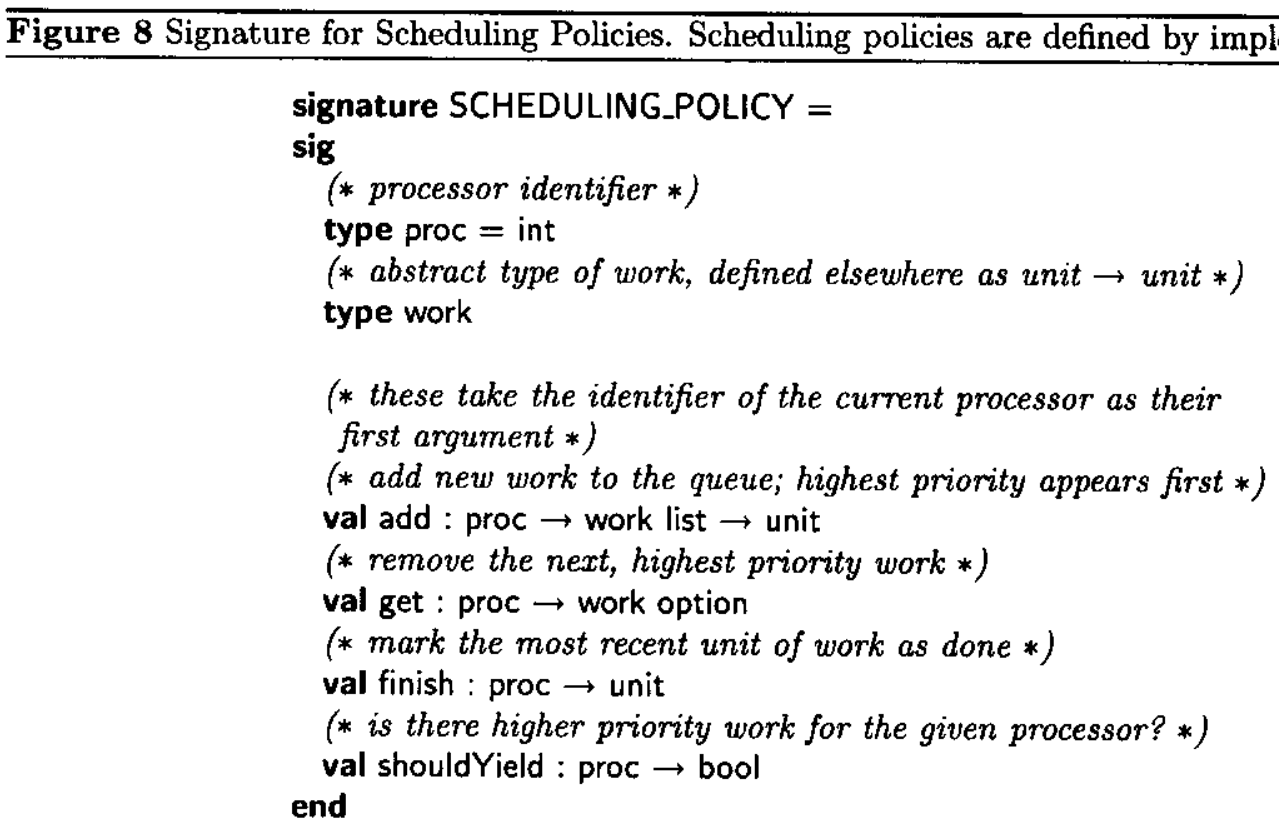

executing processors. In our initial revision, we added a global mutex around all accesses to these shared resources. We then found the hottest code paths and replaced this mutex with lighter-weight mechanisms or restructured the code to avoid synchronization altogether. In some cases, this required adding per-processor state. For example, each processor maintains a local allocation pool that it may use to satisfy allocation requests without synchronization. When the local pool is exhausted, the runtime uses an atomic compareand-swap operation to claim a portion of memory from the global pool. We were also required to make some minor changes to the compiler and standard basis library to ensure thread safety.

We have not yet addressed the issue of parallel garbage collection in our implementation. However, we believe that previous work in parallel collection for SML [Cheng and Blelloch, 2001] could be carried over in a straightforward manner.

Our extended runtime supports an additional runtime parameter that indicates how many processors to use. $^{2}$ For each processor, the runtime sets up the local processor state and invokes the main scheduling loop. The remaining parallel functionality, including the scheduling loop, is handled by a set of SML modules, described below.

\subsection{Scheduling Policies}

At the core of our parallel library is the scheduler loop. The loop is run in parallel by each processor and repeatedly executes the highest priority task that is ready to be run. It is the role of the scheduling policy to determine the highest priority task. In order to plug-and-play with different schedulers, we developed a simple signature that any scheduling policy must implement (Figure 8).

Given the purpose of scheduling policies, the functions add and get should be self-explanatory. The finish function is called once for each task removed from the queue. For many scheduling policies, finish does nothing. The final function shouldYield is used to avoid some non-trivial thread operations in cases where they are unnecessary. This operation is discussed in more detail in the description of the work-stealing scheduler below. Though we present this interface as an SML signature, we believe that this abstraction

\footnotetext{
${ }^{2}$ We envision a version that allows users to dynamically add and remove processors from the active set, but in the current implementation, this set remained fixed.
} 
would be useful for other data parallel implementations.

We include three scheduling policies in our analysis and implementation: depth-first, breadth-first, and work-stealing. (Each is between 50 and 125 lines of SML in our implementation.) Each of these policies is greedy, in that processors will not be kept idle if there are available tasks. Moreover, each permits rescheduling only at fork points and join points. These are features of the particular schedulers we study and not limitations of our framework.

Breadth-First Scheduling The breadth-first policy is the simplest policy in our implementation. It maintains a single FIFO queue and uses a global lock to serialize concurrent access to the queue. This scheduling policy is equivalent to a left-to-right, breadth-first traversal of the computation graph. It is the "fairest" of the three schedulers we implemented.

Depth-First Scheduling The parallel depth-first policy [Blelloch et al., 1999] prioritizes tasks according to a left-to-right depth-first traversal of the computation graph. Our implementation uses a single global queue and runs tasks from the front of the queue. This it not strictly a LIFO queue, however. To ensure that our implementation obeys the global left-to-right depth-first priority, the children of the leftmost task must be prioritized more highly than the children of nodes further to the right. (In a sense, priority is inherited.) To assign proper priorities, our implementation also maintains one "finger" for each processor that indicates where new tasks should be inserted into the queue [Blelloch et al., 1999]. The finish function is used to clean up any state associated with this finger.

Work-Stealing Scheduling The work-stealing policy [Blumofe and Leiserson, 1999] maintains a separate queue for each processor. Locally, each queue is maintained using a LIFO discipline. However, if one of the processors should exhaust its own queue, it randomly selects another processor to "steal" from and then removes the oldest task from that queue. In the common case, each processor only accesses its own queue, so we can use a more finely-grained synchronization mechanism than in the other two scheduling policies to serialize concurrent access.

Because a work-stealing policy favors local work, a dequeue that immediately follows an enqueue will always return the task that was enqueued. Our implementation avoids these two operations (and also avoids suspending the current thread) by always returning false as the result of shouldYield. The remainder of the parallel library checks the result of this function in cases where a dequeue will follow an enqueue.

\subsection{Parallel Library}

The lowest-level parallel interface in our library provides methods for suspending and resuming computation along with adding new tasks to the work queue. It is built as a thin wrapper around MLton's user-level thread library. ${ }^{3}$ This wrapper adds the proper calls to the scheduling policy to ensure that tasks are initiated in the proper order and finished correctly. This interface, however, is not intended for programmers. Instead, we also provide routines for parallel pairs, futures, and array manipulation based on these primitives. For example, the parallel pair construct used in our cost semantics is implemented by the following function.

$$
\begin{aligned}
& \text { (* run two functions, possibly in parallel, and return their results } \\
& \text { as a pair } * \text { ) } \\
& \text { val fork : (unit } \rightarrow \alpha) *(\text { unit } \rightarrow \beta) \rightarrow \alpha * \beta
\end{aligned}
$$

This function is implemented by (possibly) suspending the current computation and adding two new parallel tasks, one for each branch of the fork. Through the use of shared state and an atomic compare-and-swap operation, these tasks agree which of the two finished second. This task is responsible for adding a third

\footnotetext{
${ }^{3}$ MLton's thread library implements one-time continuations with functionality similar callec and throw, except that the argument to "callcc" must return another thread to switch to, and a "continuation" may only be thrown to once.
} 
task that will resume the suspended computation with the new pair. The other routines in our library are implemented in a similar manner, or by building upon functions such as fork.

\subsection{Space Profiling in MLton}

In the course of gathering our empirical results, we needed to measure space use for applications compiled with MLton. Like many garbage collectors, the MLton implementation can easily compute and report the live data after each collection and thus an approximation of the high-water mark. However, given the default behavior of the collector, there is no way to understand the accuracy of that approximation. In fact, using the collector to determine the high-water mark of space use with perfect accuracy would require a collection after every allocation or pointer update. This would be prohibitively expensive.

However, if we accept bounded errors in our measurement, we use the collector to measure this quantity with relatively little effect on performance. To measure the high-water mark of space use within a fraction $R$ of the true value, we restrict the amount of space available for new allocations as follows. At the end of each collection, given that the current high-water mark is $M$ bytes and there are currently $L$ bytes of live data in the heap, we restrict allocation so that so more than $M *(1+R)-L$ bytes will be allocated before the next collection. In the interim between collections (i.e., between measurements) the high-water mark will be no more than $M *(1+R)$ bytes. Since the collector will report at least $M$ bytes, it will achieve the desired level of accuracy.

As we report in the section on empirical results below, this technique has enabled us to measure space use with low overhead and predicable results and without any additional effort by the programmer.

\section{Empirical Results}

We performed our experiments on a four-way dual-core x86-64 machine with 32 GBs of physical RAM running version 2.6.21 of the GNU/Linux kernel. Each of the four processor chips is a $3.4 \mathrm{GHz}$ Intel Xeon, and together they provide eight independent execution units. In the remainder of this section, we will refer to each execution unit as a processor. We focus on measurements of space use, but also report on scalability. Each application is described in more detail below.

\subsection{Space Use}

For each application, we report the effect of scheduling policy and number of processors on the amount of memory required by the application. We measured the high-water mark of space use including both stacks and reachable objects in the heap. Measuring this quantity with complete accuracy would require traversing all reachable objects in the heap after every allocation and pointer mutation. Instead, we use the technique described in Section 6.4 to measure this quantity within a tunable bound. Figure 9 shows the high-water mark of space use for four of the applications in our study. Smaller values indicate better performance. We use different shapes to represent different policies: $\times$ for breadth first, + for depth-first, and $\square$ for work-stealing. Larger symbols indicate more processors were made available.

Matrix Multiplication The analysis in Section 2 (recall Figure 2(b)) predicts that the breadth-first scheduling policy uses asymptotically more space than the depth-first policy. A similar analysis predicts that breadth-first is far worse than work-stealing. Both these predictions are confirmed by the space use in our implementation, as plotted in Figure 9(a).

Sorting We implemented several sorting algorithms including quicksort, mergesort, insertion sort, and selection sort. Figure $9(\mathrm{~b})$ shows the space use of a functional implementation of quicksort where data are represented as binary trees with lists of elements at the leaves. This plot shows the behavior for the worst-case input: the input elements are given in reverse order. While we would expect quicksort to take time quadratic in the size of the input in this case, it is perhaps surprising that it also requires quadratic 
Figure 9 Space Use vs. Input Size. Each plot shows the high-water mark of space use for one of four applications. We tested three scheduling policies, depth-first $(+)$, breadth-first $(\times)$, and work-stealing $(\odot)$, with up to four processors. (Larger symbols indicate that more processors were used.) Different scheduling policies yield dramatically different performance, as discussed in the text.

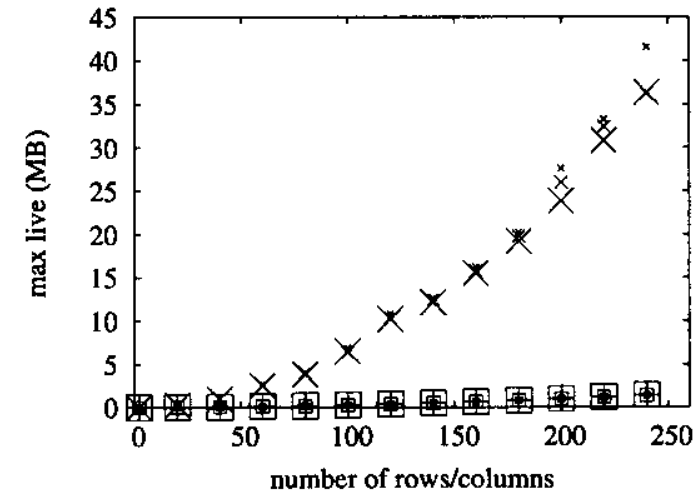

(a) matrix multiplication

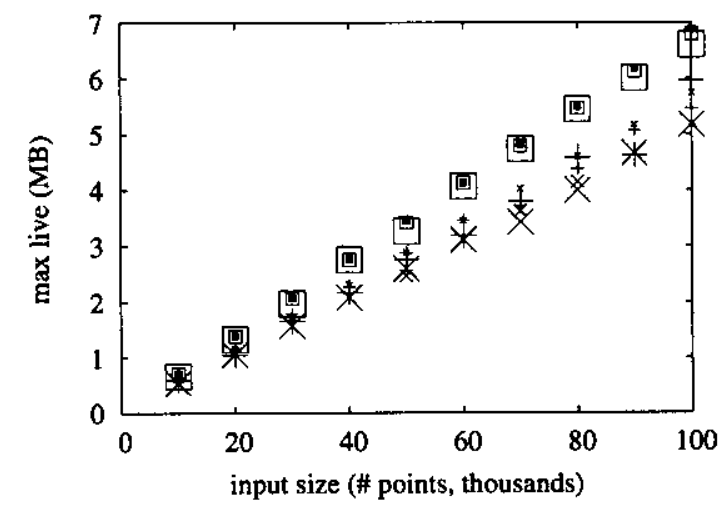

(c) quickhull

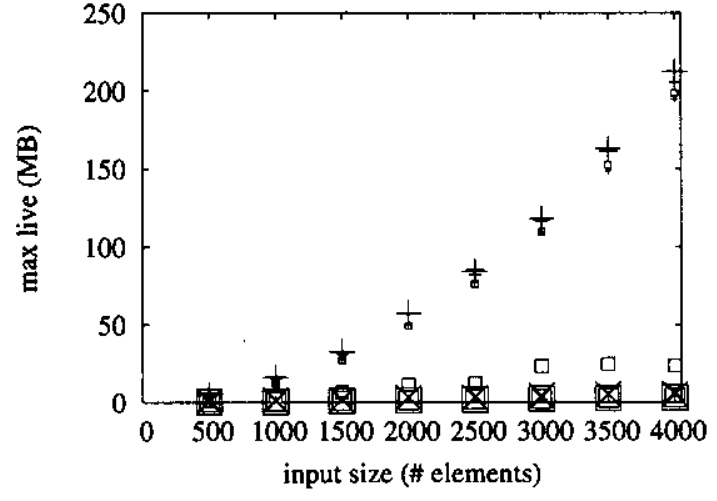

(b) quicksort

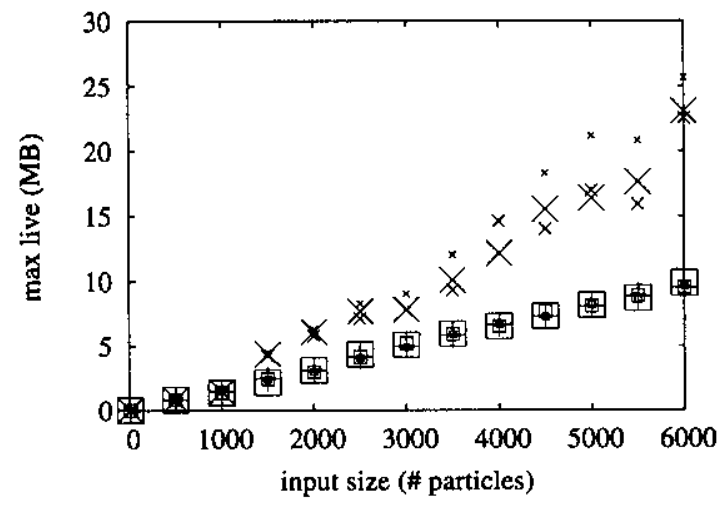

(d) Barnes-Hut simulation 
Figure 10 Space Use and Cost Graphs for Quicksort. The plot on the left (a) assigns space use at the high-water mark to parts of the source code. The summarized graphs (b) also show the point at which the program reaches this high-water mark. Both the plot and the graphs describe space use under a depth-first scheduling policy.

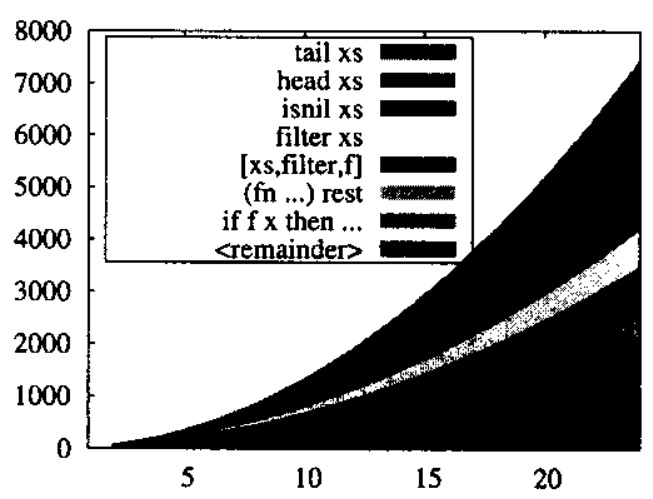

(a)

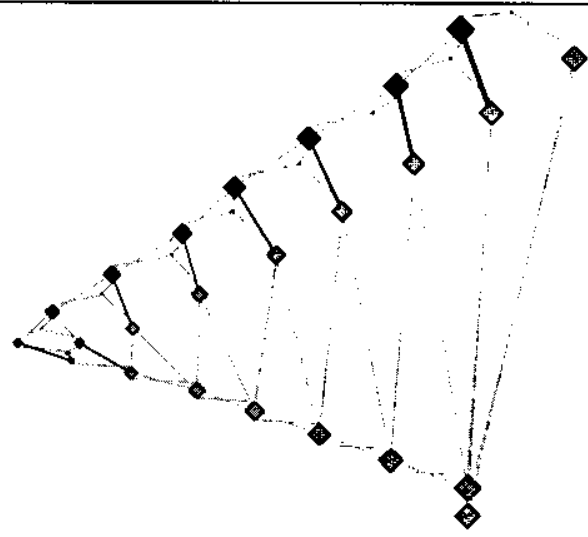

(b)

space. This behavior is also predicted by the cost semantics. The plot in Figure 10(a) shows the consumers of space for the depth-first policy at the high-water mark. The plot shows that this space is referenced by various parts of the filter function.

The graph in Figure 10(b) shows that the high-water mark for this policy occurs after the left branch of each parallel fork has executed. As expected, there are few opportunities for parallel execution with this input because at each level of the recursion, quicksort splits its input into a single element on the right and all the remaining elements on the left. However, until the partition is complete each branch on the right-hand side is still holding on to the recursive input. This analysis suggests an alternative implementation. If we introduce a join point between partitioning the elements and recursively sorting them, we can avoid the asymptotic increase in space use.

Convex Hull This application computes the convex hull in two dimensions using the quickhull algorithm. We again show results for the worst-case input: the input points are arranged in a circle and so every point in the input is also in the hull. Figure 9(c) shows the high-water mark of space use, which again matches our cost semantics-based predictions (not shown).

Unlike in the quicksort case, there is still significant available parallelism in this example. However, it is more constrained than the parallelism available in matrix multiplication. The algorithm proceeds by partitioning the point set by dividing the plane in half (in parallel) and then recursively processing each half (in parallel). Between these two phases there is a synchronization. This is shown through the widening and narrowing of the graph in Figure 11.

Nodes are colored to illustrate one point in the execution of the work-stealing scheduling policy. In this case, the work-stealing policy performs more poorly than either of the other two policies because it starts, but does not finish, computing these partitions. The point labeled " $\mathrm{A}$ " represents the code that allocates one set of points. The black lines extending to the right of this point indicate part of the program that will compute one half of a partition of these nodes. The circled nodes labeled "B" also compute half a partition, but have already completed their work and have allocated the result. At this point in time, the program is holding onto the entire original set plus half of the resulting partition. The same pattern appears for each processor. Neither of the other two scheduling policies exhibit this behavior. 
Figure 11 Cost Graphs for Quickhull. These summarized graphs show the high-water mark of space use under a work-stealing scheduling policy.

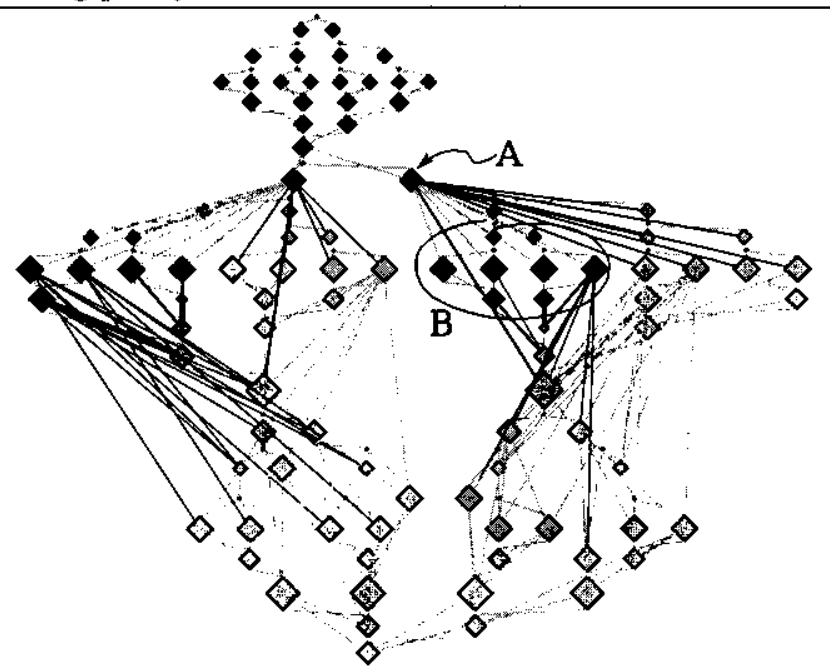

n-Body Simulation Figure 9(d) shows space use for our implementation of the Barnes-Hut simulation. This algorithm approximates the gravitational force among particles in 3-space. The force on each particle is calculated either by computing the pairwise force between two particles or by approximating the effect of a distant set of particles as a single, more massive particle. Particles are organized using an octree. This algorithm and representation are easily adapted to a parallel setting: not only can the forces on each particle be computed in parallel, but the individual components of this force can also be computed in parallel.

Like the matrix multiplication example, the breadth-first scheduling policies performs poorly due to the large amount of available parallelism and the size of the intermediate results. Though its performance is not as bad as in the multiplication example, it is still significantly worse than the other two policies.

\subsection{Overhead of Space Measurements}

Measuring space use precisely can be expensive. Using the technique described above, however, we can measure the high-water mark of space use within a fixed bound. Here, we compare the quality and cost of these measurements with those derived from a hand instrumented version. In Figure 12(a), we show space use measurements for the matrix multiplication example, but measured four different ways. All these data series use the breadth-first scheduler and one processor. The first series $(\odot)$ shows the measurements obtained when additional garbage collections are explicitly added by the programmer. The other three series show the results using the restricted allocation technique with a bound of $10 \%, 20 \%$, and $50 \%$, respectively. These bounds are shown with error bars, but only positive errors are shown (as the true high-water mark cannot be smaller than the reported value). The reported values appear at the bottom of the indicated ranges.

We take the measurements derived from the explicit garbage collection to be the most accurate measurement of memory use. (In each case, this technique reported the greatest values.) The figure shows that the restricted allocation measurements are much more accurate than we might expect. For example, the $50 \%$ bound seems to be overly conservative: the actual measurements are within $15 \%$ of the measurement derived using explicit garbage collections.

In addition to requiring less knowledge on the part of the programmer and yielding measurements with a bounded error, this technique requires less time to perform the same measurements. Figure 12(b) shows the execution time of these four instrumented versions. Values are normalized to the execution time of an uninstrumented version and shown on a logarithmic scale. 
Figure 12 Profiling Space Use. Four different measurements are shown on the left (a). On the right (b), we show the cost of obtaining these measurements. Execution times are normalized to an uninstrumented version and shown on a logarithmic scale.

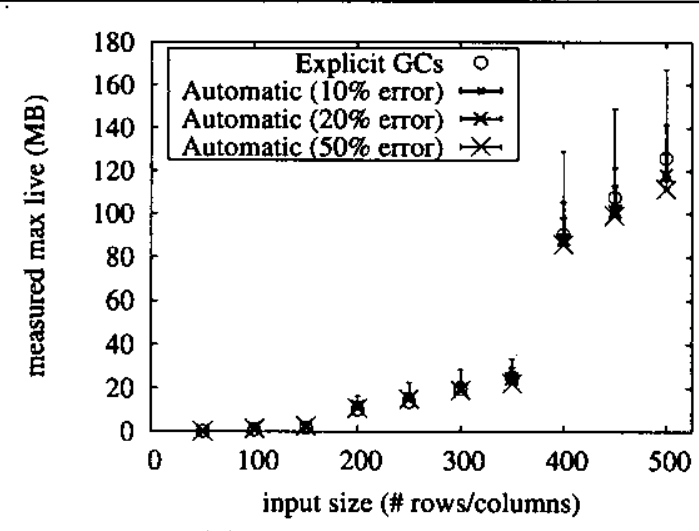

(a) measured space use

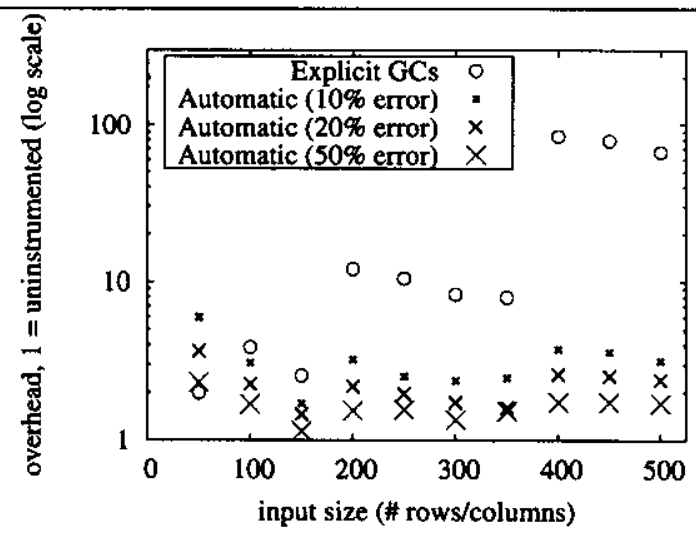

(b) instrumentation overhead

\subsection{Scalability}

As the purpose of parallelism is to improve performance, we also report on scalability. While we are still working to improve the performance of our implementation, these data should be sufficient to convince the reader that we have not "cooked" our implementation simply to match the space use predictions of our semantic profiler.

Figure 13 shows normalized parallel overhead for one to eight processors for several different applications. Parallel overhead is defined as (execution time $\times$ number of processors). We normalize this value to the execution time of the sequential version. Smaller values are better. A value of 1.0 is an ideal value indicating perfect speed-up. A value of 2.0 indicates, for example, that a four processor execution would take $50 \%$ of the time of the sequential version or that an eight processor execution would take $25 \%$ of the time of the sequential version.

In these plots, we do not include the cost of garbage collection. As we argued above, previous work has shown that garbage collection can be performed efficiently in parallel. We do, however, include overhead due to synchronization and contention for other shared resources.

Though the ideal value is 1.0 , we generally expect some overhead from parallel execution. Practically speaking, we are looking for applications (and scheduling policies) where points fall on a line with a slope close to zero. This indicates that adding additional processors will continue to improve performance at roughly the same rate.

We chose benchmarks for this set of measurements not based on interesting space use patterns, but instead by looking for applications with a reasonable potential for parallel execution. Part (a) shows overhead for a blocked version of matrix multiplication. Part (b) shows overhead for parallel mergesort on uniformly randomly distributed input. Part (c) shows the overhead of quickhull for points distributed uniformly a circle. Part (d) shows the overhead for the Barnes-Hut simulation with points distributed uniformly randomly.

\section{Discussion}

\subsection{Alternative Rules}

There are a number of design choices latent in the rules given in Figure 4. Different rules would have led to constraints that were either too strict (and unimplementable) or too lax. 
Figure 13 Parallel Scalability. These plots show parallel overhead, defined as (execution time $\times$ number of processors), normalized to the sequential execution time. Smaller values are better with the ideal being 1.0. As an example, a value of 2.0 indicates that an 8 processor execution achieves a $4 \times$ speed-up over a sequential execution, a 4 processor execution achieves a $2 \times$ speed-up, etc. Overhead does not include garbage collection.

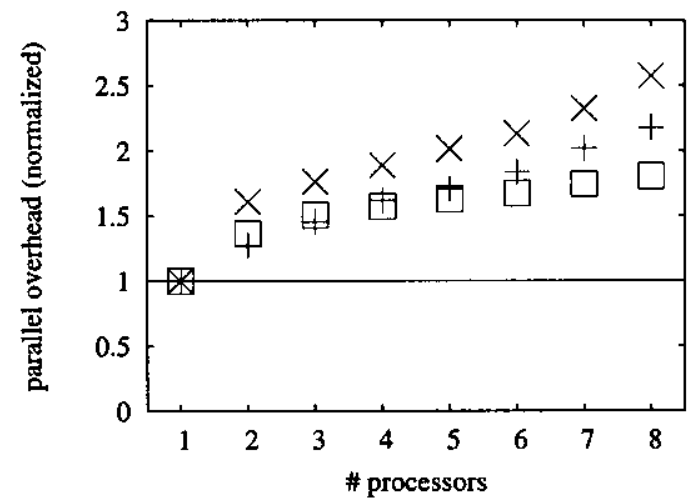

(a) matrix multiplication

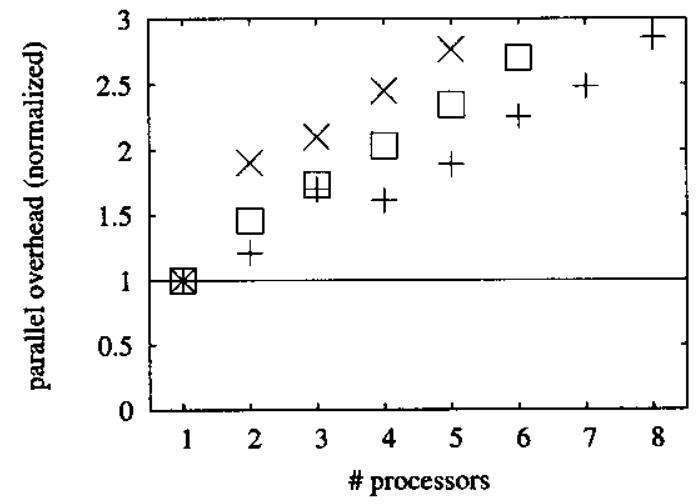

(c) quickhull

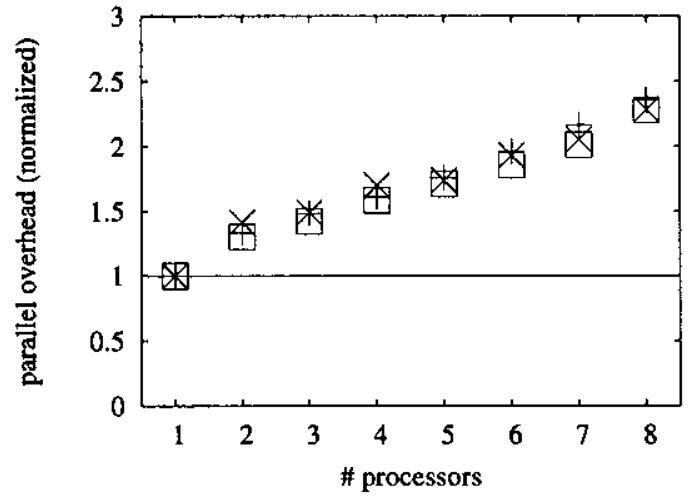

(b) mergesort

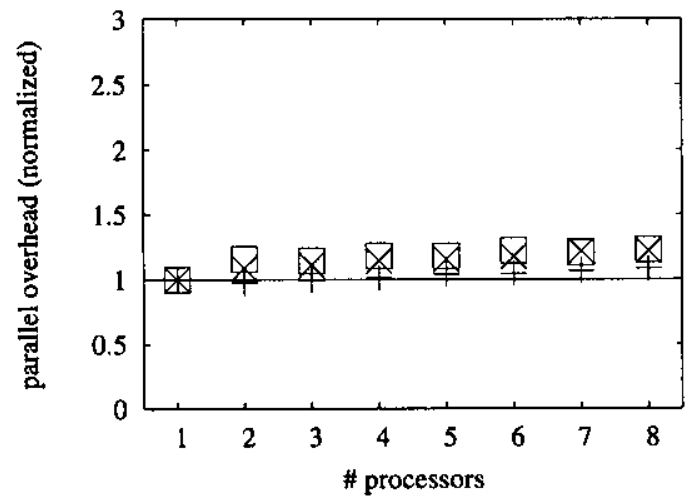

(d) Barnes-Hut simulation 
Consider as an example the following alternative rule for the evaluation of function application. The premises remain the same, and the only difference from the conclusion in Figure 4 is highlighted with a rectangle.

$$
\begin{aligned}
& e_{1} \Downarrow\left\langle f . x . e_{3}\right)^{\ell_{1}} ; g_{1} ; h_{1} \quad e_{2} \Downarrow v_{2} ; g_{2} ; h_{2} \quad \cdots \\
& \hline e_{1} e_{2} \Downarrow v_{3} ; g_{1} \oplus g_{2} \oplus g_{3} \oplus[n] ; ; \\
& h_{1} \cup h_{2} \cup h_{3} \cup\left\{\left(n, \ell_{1}\right),\left(n, \operatorname{loc}\left(v_{2}\right)\right)\right\}
\end{aligned}
$$

This rule yields the same extensional result as the version given in Figure 4, but it admits more implementations. Recall that the heap edges $\left(n, \ell_{1}\right)$ and $\left(n, \operatorname{loc}\left(v_{2}\right)\right)$ represent possible last uses of the function and its argument, respectively. This variation of the rule moves those dependencies until after the evaluation of the function body.

This rule allows implementations to preserve these values, even in the case where they are not used during the function application or in subsequent evaluation. This includes the case where these values are bound to variables that do not appear in the program text of the function body or after the application. This is precisely the constraint described by Shao and Appel [1994] as "safe-for-space."

In contrast, the original version of this rule requires that these values be considered for reclamation by a garbage collector as soon as the function is applied. Note, however, that the semantics does not specify how the implementation makes these values available for reclamation: it is left to the implementer to determine whether this is achieved through closure conversion, by clearing slots in the stack frame, or by some other means.

As this example suggests, there is some leeway in the choice of the semantics itself. Our goal was to find a semantics that describes a set of common implementation techniques. When they fail to align, our experience suggests that either the semantics or the implementation can be adjusted to allow them to fit together.

\subsection{Program Optimizations}

In the course of validating our work, we discovered one example where we were required to change the implementation. We came across an example where our implementation failed to distinguish two scheduling policies as our profiler had predicted. Specifically, instead of one policy leading to linear use of space and the other to quadratic use of space, the program required quadratic space under both policies.

Some investigation revealed that the problem was not in our semantics or our parallel implementation, but in an existing optimization in MLton: reference flattening. Analogously to tuple flattening, references that appear in a heap-allocated data structure (e.g. a record) may be flattened or treated as a mutable field within that structure. At run time, such a reference is represented as a pointer to the containing structure. Accesses to the reference must compute an offset from that pointer.

This optimization can save some space by eliminating the memory cell associated with the reference. However, it can also increase the use of space. As the reference is represented as a pointer to the entire structure, all of the elements of the structure will be reachable anywhere the reference is reachable. If the reference would have outlived its enclosing structure then flattening will extend the lifetime of the other components.

To avoid asymptotically increasing the use of space, MLton uses the types of the other components of the structure to conservatively estimate the size of each component. If any component could be of unbounded size then the reference is not flattened. The problem was that this analysis was not sufficiently conservative in its treatment of recursive types. Though a single value of a recursive type will only require bounded space, an unbounded number of these values may be reachable through a single pointer. Based on our reporting, this problem has been corrected in a recent version in the MLton source repository.

\subsection{Nested Parallelism}

Another form of "flattening" appears in implementations of nested parallelism such as NESL [Blelloch et al., 1994] or Nepal [Chakravarty and Keller, 2000]. Here, flattening refers to a compilation technique where 
Figure 14 Labeled Expressions. Expressions are labeled according to their position in an abstract syntax tree. Any labeling that assigns a unique label to each sub-expression would be sufficient to force a SIMDstyle parallel execution. However, for the purposes of emulating the behavior of flattened nested parallelism, we assume a set of labels derived from sequences of integers. We also add array operations to the source language.

$$
\begin{aligned}
& \text { (labels) } \quad \alpha, \beta \quad:=\epsilon \mid \alpha, n \\
& \text { (expressions) } \quad \text { e }::=\ldots \mid \text { idx } e\left|\operatorname{map} e_{1} e_{2}\right| \alpha: e \\
& \text { (values) } \quad v::=\ldots\left|\left\langle v_{1}, \ldots, v_{k}\right\rangle^{\ell}\right| \alpha: v \\
& \overline{\alpha \triangleright x \leadsto \alpha: x} \quad \overline{\alpha \triangleright \text { true } \leadsto \alpha: \text { true }} \\
& \frac{\alpha, 1 \triangleright e \rightsquigarrow e^{\prime}}{\alpha \triangleright \text { fun } f(x)=e \rightsquigarrow \alpha: \text { fun } f(x)=e^{\prime}} \quad \frac{\alpha, 1 \triangleright e_{1} \rightsquigarrow e_{1}^{\prime} \quad \alpha, 2 \triangleright e_{2} \rightsquigarrow e_{2}^{\prime}}{\alpha \triangleright e_{1} e_{2} \rightsquigarrow \alpha:\left(e_{1}^{\prime} e_{2}^{\prime}\right)} \\
& \frac{\alpha, 1 \triangleright e_{1} \leadsto e_{1}^{\prime} \quad \alpha, 2 \triangleright e_{2} \leadsto e_{2}^{\prime} \quad \alpha, 3 \triangleright e_{3} \leadsto e_{3}^{\prime}}{\alpha \triangleright \text { if } e_{1} \text { then } e_{2} \text { else } e_{3} \leadsto \alpha \text { if } e_{1}^{\prime} \text { then } e_{2}^{\prime} \text { else } e_{3}^{\prime}} \\
& \frac{\alpha, 1 \triangleright e \rightsquigarrow e^{\prime}}{\alpha \triangleright \text { idx } e \rightsquigarrow \alpha: \operatorname{idx} e^{\prime}} \quad \frac{\alpha, 1 \triangleright e_{1} \leadsto e_{1}^{\prime} \alpha, 2 \triangleright e_{2} \rightsquigarrow e_{2}^{\prime}}{\alpha \triangleright \operatorname{map} e_{1} e_{2} \leadsto \alpha \text { :map } e_{1}^{\prime} e_{2}^{\prime}}
\end{aligned}
$$

by nested parallelism (i.e., parallel tasks may recursively spawn new parallel tasks) is transformed into flat. parallelism (em i.e., parallel tasks are spawned only at the top level). This automatically balances work by exposing all available parallelism at the same time.

Previous work has shown that nested parallelism can be implemented efficiently by transforming nested structures into flat arrays of primitive values [Blelloch and Sabot, 1990, Suciu and Tannen, 1994, Au et al., 1997, Chakravarty and Keller, 2000]. This opens opportunities to take advantage of hardware support for parallelism through SIMD (single instruction, multiple data) instructions. Such parallelism is currently supported by multimedia extensions to many general-purpose processors as well as by special-purpose graphics and vector processors. In the remainder of this section, we will restrict our use the term data parallelism to describe the sort of parallelism made available by these types of hardware (where all steps taken in parallel must perform the same primitive operation). We also extend of our language with immutable arrays and operations over these arrays. For example, arrays are created using idx (which given an integer $n$, creates an array of length $n$ which each element is equal to its index).

Though flattening (or vectorizing) is often described as a compiler transformation, it also can be viewed as a set of constraints on what parts of a program can be executed in parallel, or in other words, as a schedule. Instead of flattening the code, we now describe a variant of our parallel transition semantics that captures the behavior of a flattened program. This will allow us to reuse the other machinery we have developed to reason about space use. It also allows us to express alternative implementation strategies that have better worse-case performance.

In most cases, the behavior of flattened code can be easily integrated into our framework. Some care must be taken with forms of expressions that allow branching, including conditional expressions. These forms of expressions (when they appear in the context of parallel evaluation) are sequentialized by the flattening transformation.

We refine our transition semantics in two stages. First, we constrain our semantics to allow only the sorts of parallelism implementable by SIMD hardware. We accomplish this using a version of the labeled lambdacalculus [Lévy, 1978, Abadi et al., 1996]. Second, we impose further restrictions, including a breadth-first schedule, to precisely emulate the effects of the flattening transformation.

Data Parallelism Via Labeling To capture the behavior of a SIMD-style parallel implementation, we must constrain the sorts of schedules that we allow in our analysis and implementation. When a function is mapped across an array, we must ensure that any conditional sub-expressions of that function are properly 
Figure 15 Breadth-First Labeled Transition Semantics. We describe the behavior of flattened nested parallelism using a family of transition relations, indexed by labels $\alpha$. Notably, the expressions here are not flattened, but the behavior of flattened code is emulated by the constrained semantics: primitive transitions may only be applied if the label of the expression matches the index of the relation.

$e \stackrel{\text { bf-dp }}{\longmapsto} e^{\prime}$

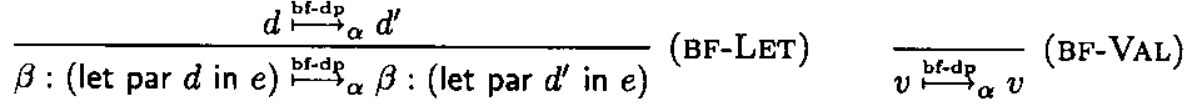

$$
\begin{aligned}
& \frac{e \longrightarrow_{\alpha} e^{\prime}}{\alpha: e \stackrel{\text { bf-dp }}{\longrightarrow} \alpha: e^{\prime}} \text { (BF-PRIM) } \frac{\alpha \neq \beta}{\beta: e \stackrel{\text { bf-dp }}{\longrightarrow} \beta: e} \text { (BF-WAIT) }
\end{aligned}
$$

$d \stackrel{\text { bf-dp }}{\longrightarrow} d^{\prime}$

$$
\frac{e \stackrel{\text { bf-dp }}{\longrightarrow} e^{\prime}}{x=e \stackrel{\text { bf-dp }}{\longrightarrow} \alpha=e^{\prime}} \text { (BF-LEAF) } \quad \frac{d_{1} \stackrel{\text { bf-dp }}{\longmapsto} d_{1}^{\prime} \quad d_{2} \stackrel{\text { bf-dp }}{\longmapsto} d_{2}^{\prime}}{d_{1} \text { and } d_{2} \stackrel{\text { bf-dp }}{\longrightarrow} d_{\alpha}^{\prime} \text { and } d_{2}^{\prime}} \text { (BF-BRANCH) }
$$

sequentialized. To this end, we define labeled expressions as shown in Figure 14. Each expression is given a label using the following judgment.

$$
\alpha \triangleright e \leadsto e^{\prime}
$$

This judgment is read, assuming the current context is a label $\alpha$ then expression $e$ is rewritten as labeled expression $e^{\prime}$. This judgment rewrites source terms into their labeled counterparts. While these labels initially correspond to the tree address of each node in the abstract syntax tree, this property will not be maintained by evaluation. Note that each branch of a conditional expression is labeled starting with a different prefix.

With labeled expressions, we can now succinctly state the following constraint: data parallel implementations only execute expressions in parallel if those expressions have the same label. Since each label comes from a unique sub-expression of the original term, duplicate labels will only appear when they are introduced by the evaluation of terms such as map, as discussed below.

In general, any labeling that assigns a unique label to each sub-expression will be sufficient to enforce a SIMD-style parallel execution. However, we use sequences of integers as labels to enable us to capture the effect of the flattening transformation. In particular, we will use a lexicographic ordering (based on the natural ordering of integers) to ensure that proper sequencing of conditional expressions.

Flattening $=$ Labeling + Breadth-First Parallelism We now define a transition semantics on labeled expressions that emulates the behavior of the flattened versions of these same expressions, as shown in Figure 15. This semantics differs from the non-deterministic transition semantics in Section 6 in two ways. First, it requires that all possible parallelism be exploited. That is, if two sub-expressions can take a primitive step in parallel, then this semantics will always apply both primitive steps. This yields a deterministic, breadth-first schedule.

Second, this semantics is defined as a family of transition relations indexed by labels. Each relation in this family will only apply primitive transitions to expressions with the corresponding label. The rule BF-WAIT is applied to all other expressions, leaving them unchanged. The remaining rules either ignore labels or pass them through unchanged. 
Figure 16 Labeled Primitive Transitions. As for the breadth-first data parallel semantics, this semantics is a family of relations indexed by label. Most primitive transitions remain unchanged. The two rules shown here are the exceptions: map replicates its label and application freshens the labels in the body of the function using label-indexed substitution.

$$
\begin{aligned}
& \overline{\operatorname{map} v\left\langle v_{1}, \ldots, v_{n}\right\rangle^{\ell} \longrightarrow \alpha}\left(\mathrm{P}_{\alpha}-\mathrm{MAP}\right) \\
& \text { let par } x_{1}=\alpha:\left(v v_{1}\right) \text { and } \ldots \\
& \text { and } x_{n}=\alpha:\left(v v_{n}\right) \\
& \quad \text { in }\left[x_{1}, \ldots, x_{n}\right] \\
& \overline{\langle f . x . e\rangle^{\ell} v_{2} \longrightarrow \alpha\left[\langle f . x . e\rangle^{\ell} / f\right]^{\alpha}\left[v_{2} / x\right]^{\alpha} e}\left(\mathrm{P}_{\alpha} \text {-APPBETA }\right)
\end{aligned}
$$

The complete evaluation of an expression is defined by a sequence of expressions $e_{0}, \ldots, e_{k}$ and an ordered sequence of labels $\alpha_{0}, \ldots, \alpha_{k-1}$, such that for all $i \in[0, k)$,

$$
e_{i} \stackrel{\text { bfodp }}{\longmapsto} \alpha_{i} e_{i+1}
$$

A lexicographic ordering of labels ensures that the left branch of a conditional expression is always completely evaluated before the right branch is initiated. Without this ordering, this transition semantics would allow additional schedules that are not implemented by a flattening transformation. For example, without this constraint, an implementation might interleave evaluation of the two branches of a parallel conditional expression, rather than exhaustively executing the left branch first.

This parallel transition semantics makes use of an indexed primitive transition relation. In most cases, this index is ignored. The two exceptions are shown in Figure 16. First, when a function is mapped across an array, each application is given the same label. Second, when a function is applied in an ordinary context, the index of the relation is used when substituting the argument into the body of the function. The purpose of this substitution is to specialize the function body to the calling context. In particular, whenever a value is substituted into a labeled expression, that label of that expression is rewritten as shown by the following definition (where "," is overloaded here to mean the concatenation of sequences).

$$
[v / x]^{\alpha}(\beta: e)=(\alpha, \beta):\left([v / x]^{\alpha} e\right)
$$

The remainder of the label-indexed substitution rules follow from the standard definition of capture-avoiding substitution.

If a function is bound to a variable that occurs more than once in the source code, then the body of that function will be specialized to each context in which it is applied. If a function is applied in both branches of a conditional expression, each instance will be relabeled with a different set of labels (preventing them from executing in parallel). On the other hand, when a function body is duplicated as a result of map, every occurrence of the body will be specialized with the same label. As an example, consider the following expression.

$$
\text { map (fun } f(x)=\text { if even } x \text { then } x \operatorname{div} 2 \text { else }(x+1) \operatorname{div} 2 \text { ) (idx 10) }
$$

A flattening compiler will lift the scalar integer operations (e.g., even, div, + ) to the analogous vector versions so that within each branch, these operations may be performed in parallel. The branches of the conditional expression, however, will be evaluated serially. The flattened code will collect together all the elements from the original array that satisfy the predicate and (separately) those that do not. Next, it will execute the first branch, exploiting whatever parallelism is available, followed by the second branch. (From the point of view of a flattening compiler, both branches of the conditional are taken, but different elements are passed to each branch.) Even though div operations appear in both branches, they will not be evaluated in parallel. The labeling described in this section enforces this constraint by giving different labels to the opcrations that appear in each branch. 
Though we omit a detailed explanation, a sequence of labeled transitions can be related to labelconstrained schedule of a cost graph, as well as to the execution of the flattened form of the original expression.

This characterization of flattening as a form of breadth-first scheduling agrees with our experience in NESL: examples such as matrix multiplication required so much space in NESL that they were unrunnable for even moderately sized inputs, requiring the user to scale back parallelism explicitly. These space use problems in NESL are an important part of the motivation for the current work.

One advantage of considering the flattening transformation as a schedule is that we have not lost the original structure of the expression. We can therefore define alternative data parallel implementations, such as one that respects labeling but more closely follows a parallel depth-first schedule. Defining such an implementation within our semantic framework is straightforward. We leave as future work a version of flattening that yields programs with similar performance.

\section{Related Work}

Parallelism and Concurrency Many researchers have studied how to improve performance by exploiting the parallelism implicit in side-effect free languages. This includes work on data-flow languages (e.g., Arvind et al. [1989]), lazy parallel functional languages (e.g., Aditya et al. [1995]), and nested data parallel languages (e.g., Blelloch et al. [1994], Chakravarty and Keller [2000]). Like these languages, we provide a deterministic semantics and rely on the language implementation to spawn new tasks and synchronize on results. Unlike languages such as Concurrent ML [Reppy, 1999] or JoCaml [Conchon and Fessant, 1999], we provide no explicit concurrency constructs to the programmer. Manticore [Fluet et al., 2007] subsumes both paradigms and provides for both implicit parallelism and explicit concurrency.

Profiling In their seminal work on space profiling for lazy functional programs, Runciman and Wakeling [1993a] demonstrate the use of a profiler to reduce the space use of a functional program by more than two orders of magnitude. Like the current work, they measure space use by looking at live data in the heap. However, their tool is tied to a particular sequential implementation. Sansom and Peyton Jones $[1993,1995]$ extend this work with a notion of "cost center" that enables the programmer to designate how resource use is attributed to different parts of the source program.

There has been a series of works on profiling methods and tools for parallel functional programs [Hammond and Peyton Jones, 1992, Runciman and Wakeling, 1993b, Hammond et al., 1995, Charles and Runciman, 1998]. This work focuses on the overhead of parallel execution instead of how different parallel implementations affect the performance of the application. None of this work measures how different scheduling policies affect the space use of applications.

Scheduling Scheduling policies and their effects on space use have been studied extensively in the algorithms community (e.g., Blumofe and Leiserson 1998, Blelloch et al. 1999). Our representation of parallel tasks as directed graphs is inspired by this work. However, we use these graphs as part of a formal semantics rather than simply an abstract model of computation.

Most implementations of data parallel languages have provided only a single scheduling policy that is either left unspecified or fixed as part of a compilation technique. In contrast, Fluet et al. [2007] make scheduling policies explicit using a coordination language. It would be interesting to see how our cost semantics could serve as a specification for schedulers written in this language.

Evaluation strategies [Trinder et al., 1998] enable the programmer to explicitly control the parallel evaluation structure (e.g., divide-and-conquer). Like much of the work on profiling parallel functional programs, this focuses on when to spawn to parallel tasks and how much work to perform in each task (i.e., granularity) instead of the order in which parallel tasks are evaluated.

In the course of their profiling work, Hammond and Peyton Jones [1992] considered two different scheduling policies. While one uses a LIFO strategy and the other FIFO, both use an evaluation strategy that shares many attributes with a work-stealing policy. These authors found that for their implementation, the 
choice of policy did not usually affect the running time of programs, with the exception in the case where they also throttled the creation of new parallel threads. In this case, the LIFO scheme gave better results. Except for the size of the thread pool itself, they did not consider the effect of policy on space use.

Cost Semantics A cost semantics was first used as a basis for profiling by Sansom and Peyton Jones [1995]. They describe a method of profiling both time and space for programs written in a sequential, lazy language. As in our work, they use the cost semantics as a formal specification of performance. However, they use the semantics only as guide for their profiler: profiling results are derived from an instrumented version of the actual language implementation.

A cost semantics similar to the one used here was introduced by Blelloch and Greiner [1996]. That work gave an upper bound on space use and assumed a fixed scheduling policy (depth-first). Our semantics extends this work by adding heap edges to the cost associated with each closed program. This enables us to reason about different scheduling policies and attribute space use to different parts of the program.

Lazy, purely functional programs can be evaluated in many different ways, and different strategies for evaluation can yield wildly different performance results. Ennals [2004] uses a cost semantics to compare the work performed by a range of sequential evaluation strategies, ranging from lazy to eager. Like the current work, he also uses cost graphs with distinguished types of edges, though his edges serve different purposes. He does not formalize the use of space by these different strategies. Likewise, program transformations that change the order of evaluation can also affect performance. Gustavsson and Sands [1999] give a semantic definition of what it means for a transformation to be "safe-for-space" [Shao and Appel, 1994]. They provide several laws to help prove that a given transformation does not asymptotically increase the space usage of sequential, call-by-need programs.

Jay et al. [1997] describe a static framework for reasoning about the costs of parallel execution using a monadic language. Static cost models have also been used to automatically choose a parallel implementation at compile-time based on hardware performance parameters [Hammond et al., 2003] and to inform the granularity of scheduling [Loidl and Hammond, 1996]. This work complements ours in that it focuses on how the sizes of program data structures affect parallel execution (e.g., through communication costs), rather than how different execution models affect the use of space at a given point in time.

\section{Conclusion}

We have described and demonstrated the use of a semantic space profiler for parallel functional programs. One beauty of functional programming is that it isolates programmers from gritty details of the inplementation and the target architecture, whether that architecture is sequential or parallel. However, when profiling functional programs, and especially parallel functional programs, there is a tension between providing information that relates to the source code and information that accurately reflects the implementation. In our profiling framework, a cost semantics plays a critical role in balancing that tradeoff.

We have focused on using our framework to measure and reason about the performance effects of different scheduling policies. One possible direction for future work is to study other important aspects of a parallel implementation such as task granularity. We believe there is a natural way to fit this within our framework, by viewing task granularity as an aspect of scheduling policy.

We invite readers to download and experiment with our prototype implementation, available from the first author's website ${ }^{4}$ or the shared-heap-multicore branch of the MLton repository.

\section{References}

Martín Abadi, Butler Lampson, and Jean-Jacques Lévy. Analysis and caching of dependencies. In ICFP '96: Proceedings of the first ACM SIGPLAN international conference on Functional programming, pages 83-91, New York, NY, USA, 1996. ACM Press.

\footnotetext{
${ }^{4}$ http://wrw.cs.cmu.edu/ $\sim$ spoons/parallel/
} 
Shail Aditya, Arvind, Jan-Willem Maessen, and Lennart Augustsson. Semantics of pH: A parallel dialect of Haskell. Technical Report Computation Structures Group Memo 377-1, MIT, June 1995.

Andrew W. Appel, Bruce Duba, and David B. MacQueen. Profiling in the presence of optimization and garbage collection. Technical Report CS-TR-197-88, Princeton University, November 1988.

Arvind, Rishiyur S. Nikhil, and Keshav K. Pingali. I-structures: data structures for parallel computing. ACM Trans. Program. Lang. Syst., 11(4):598-632, 1989.

K. T. P. Au, M. M. T. Chakravarty, J. Darlington, Y. Guo, S. Jähnichen, M. Köhler, G. Keller, W. Pfannenstiel, and M. Simons. Enlarging the scope of vector-based computations: Extending Fortran 90 by nested data parallelism. In Advances in Parallel and Distributed Computing Conference (APDC), page 66, Washington, DC, USA, 1997. IEEE Computer Society.

Guy Blelloch, Phil Gibbons, and Yossi Matias. Provably efficient scheduling for languages with fine-grained parallelism. J. of the $A C M, 46(2): 281-321,1999$.

Guy E. Blelloch and John Greiner. A provable time and space efficient implementation of nesl. In $A C M$ SIGPLAN International Conference on Functional Programming, pages 213-225, May 1996.

Guy E. Blelloch and Gary W. Sabot. Compiling collection-oriented languages onto massively parallel computers. J. Parallel Distrib. Comput., 8(2):119-134, 1990.

Guy E. Blelloch, Jonathan C. Hardwick, Jay Sipelstein, Marco Zagha, and Siddhartha Chatterjee. Implementation of a portable nested data-parallel language. J. Parallel Distrib. Comput., 21(1):4-14, 1994.

Robert D. Blumofe and Charles E. Leiserson. Space-efficient scheduling of multithreaded computations. SIAM J. of Comp., 27(1):202-229, 1998.

Robert D. Blumofe and Charles E. Leiserson. Scheduling multithreaded computations by work stealing. $J$. of the $A C M, 46(5): 720-748,1999$.

Manuel M. T. Chakravarty and Gabriele Keller. More types for nested data parallel programming. In ICFP '00: Proceedings of the fifth ACM SIGPLAN international conference on Functional programming, pages 94-105, New York, NY, USA, 2000. ACM Press.

Nathan Charles and Colin Runciman. An interactive approach to profiling parallel functional programs. In Implementation of Functional Languages, 10th International Workshop, IFL'98, London, UK, September 9-11, Selected Papers, pages 20-37, 1998.

Perry Cheng and Guy E. Blelloch. A parallel, real-time garbage collector. SIGPLAN Not., 36(5):125-136, 2001.

Silvain Conchon and Fabrice Le Fessant. Jocaml: Mobile agents for objective-caml. In ASAMA 'g9: Proceedings of the First International Symposium on Agent Systems and Applications Third International Symposium on Mobile Agents, page 22, Washington, DC, USA, 1999. IEEE Computer Society.

Robert Ennals. Adaptive Evaluation of Non-Strict Programs. PhD thesis, University of Cambridge, 2004.

Matthew Fluet, Nic Ford, Mike Rainey, John Reppy, Adam Shaw, and Yingqi Xiao. Status report: the manticore project. In $M L$ '07: Proceedings of the 2007 workshop on Workshop on $M L$, pages 15-24, New York, NY, USA, 2007. ACM.

Jörgen Gustavsson and David Sands. A foundation for space-safe transformations of call-by-need programs. In Proceedings of Workshop on Higher Order Operational Techniques in Semantics, number volume 26 of Electronic Notes in Theoretical Computer Science, September 1999. 
Kevin Hammond and Simon L. Peyton Jones. Profiling scheduling strategies on the GRIP multiprocessor. In International. Workshop on the Parallel Implementation of Functional Languages, pages 73-98, RWTH Aachen, Germany, September 1992.

Kevin Hammond, Hans-Wolfgang Loidl, and Andrew S. Partridge. Visualising Granularity in Parallel Programs: A Graphical Winnowing System for Haskell. In A. P. Wim Böhm and John T. Feo, editors, HPFC'95 - High Performance Functional Computing, pages 208-221, 1995.

Kevin Hammond, Jost Berthold, and Rita Loogen. Automatic skeletons in template haskell. Parallel Processing Letters, 13(3):413-424, September 2003.

Gérard Huet. Confiuent reductions: Abstract properties and applications to term rewriting systems: Abstract properties and applications to term rewriting systems. J. ACM, 27(4):797-821, 1980.

C. Barry Jay, Murray Cole, M. Sekanina, and Paul Steckler. A monadic calculus for parallel costing of a functional language of arrays. In Euro-Par '97: Proceedings of the Third International Euro-Par Conference on Parallel Processing, pages 650-661, London, UK, 1997. Springer-Verlag.

Peter J. Landin. The mechanical evaluation of expressions. Computer Journal, 6, Jan 1964.

Jean-Jacques Lévy. Reductions Correctes et Optimales dans le Lambda Calcul. PhD thesis, University of Paris 7, 1978.

Hans-Wolfgang Loidl and Kevin Hammond. A sized time system for a parallel functional language. In Proceedings of the Glasgow Workshop on Functional Programming, Ullapool, Scotland, July 1996.

Robin Milner, Mads Tofte, Robert Harper, and David Macqueen. The Definition of Standard ML (Revised). MIT Press, Cambridge, MA, USA, 1997.

John H. Reppy. Concurrent programming in ML. Cambridge University Press, New York, NY, USA, 1999.

Niklas Röjemo and Colin Runciman. Lag, drag, void and use-heap profiling and space-efficient compilation revisited. SIGPLAN Not., 31(6):34-41, 1996.

Colin Runciman and David Wakeling. Heap profiling of lazy functional programs. J. Funct. Program., 3(2): 217-245, 1993a.

Colin Runciman and David Wakeling. Profiling Parallel Functional Computations (Without Parallel Machines). In Functional Programming, Glasgow '93, pages 236-251. Springer-Verlag, 1993b.

Patrick M. Sansom and Simon L. Peyton Jones. Profiling lazy functional programs. In Proceedings of the 1992 Glasgow Workshop on Functional Programming, pages 227-239, London, UK, 1993. Springer-Verlag.

Patrick M. Sansom and Simon L. Peyton Jones. Time and space profiling for non-strict, higher-order functional languages. In POPL '95: Proceedings of the 22nd ACM SIGPLAN-SIGACT symposium on Principles of programming languages, pages 355-366, New York, NY, USA, 1995. ACM.

Zhong Shao and Andrew W. Appel. Space-efficient closure representations. In LFP '94: Proceedings of the 1994 ACM conference on LISP and functional programming, pages 150-161, New York, NY, USA, 1994. ACM Press.

Dan Suciu and Val Tannen. Efficient compilation of high-level data parallel algorithms. In SPAA '94: Proceedings of the sixth annual ACM symposium on Parallel algorithms and architectures, pages 57--66, New York, NY, USA, 1994. ACM Press.

Philip W. Trinder, Kevin Hammond, Hans-Wolfgang Loidl, and Simon L. Peyton Jones. Algorithm + Strategy = Parallelism. J. of Functional Programming, 8(1):23-60, January 1998.

Stephen Weeks. Whole-program compilation in mlton. In $M L$ '06: Proceedings of the 2006 workshop on $M L$, page 1, New York, NY, USA, 2006. ACM. 


\section{A Definitions}

\section{A.1 Locations}

The location of a value is the outermost location of that value and serves to uniquely identify that value. The locations of an expression are the locations of any values that appear in that expression.

$$
\begin{array}{ll}
\operatorname{loc}\left(\langle f . x . e\rangle^{\ell}\right) & =\ell \\
\operatorname{loc}\left(\left\langle v_{1}, v_{2}\right\rangle^{\ell}\right) & =\ell \\
\operatorname{loc}\left(\text { true }^{\ell}\right) & =\ell \\
\operatorname{loc}\left(\text { false }^{\ell}\right) & =\ell \\
\operatorname{locs}(\text { fun } f(x)=e) & =\operatorname{locs}(e) \\
\operatorname{locs}\left(e_{1} e_{2}\right) & =\operatorname{locs}\left(e_{1}\right) \cup \operatorname{locs}\left(e_{2}\right) \\
\operatorname{locs}\left(\left\{e_{1}, e_{2}\right\}\right) & =\operatorname{locs}\left(e_{1}\right) \cup \operatorname{locs}\left(e_{2}\right) \\
\operatorname{locs}(\# i e) & =\operatorname{locs}(e) \\
\operatorname{locs}(\text { true }) & =\emptyset \\
\operatorname{locs}(\text { false }) & =\emptyset \\
\operatorname{locs}\left(\text { if } e_{1} \text { then } e_{2} \text { else } e_{3}\right) & =\operatorname{locs}\left(e_{1}\right) \cup \operatorname{locs}\left(e_{2}\right) \cup \operatorname{locs}\left(e_{3}\right)
\end{array}
$$

\section{A.2 Substitution}

Substitution, as used in Sections 3 and 4 , is a standard capture-avoiding substitution.

$$
\begin{array}{lll}
{[v / x] x} & = & \\
{[v / x] y} & = & y \\
{[v / x] c} & = & c \\
{[v / x](\text { fun } f(y)=e)} & = & \text { fun } f(y)=([v / x] e) \\
{[v / x]\left(e_{1} e_{2}\right)} & = & \left([v / x] e_{1}\right)\left([v / x] e_{2}\right) \\
{[v / x]\left\{e_{1}, e_{2}\right\}} & = & \text { if } \left.x \notin\{v / x] e_{1},[v / x] e_{2}\right\} \\
[v / x](\# i)) & =\# i([v / x] e) \\
{[v / x](\text { let par } d \text { in } e)} & =\text { let par }[v / x] d \text { in }[v / x] e \\
{[v / x](x=e)} & = & x=[v / x] e \\
{[v / x]\left(d_{1} \text { and } d_{2}\right)} & = & {[v / x] d_{1} \text { and }[v / x] d_{2}} \\
& &
\end{array}
$$

\section{B Proofs}

\section{B.1 Confluence}

Theorem 1 (Confluence). If $e \stackrel{\text { nd }}{\longrightarrow} e^{\prime}$ and $e \stackrel{n d}{\longmapsto} e^{\prime \prime}$ then there exists an expression $e^{\prime \prime \prime}$ such that $e^{\prime} \stackrel{\text { nd }}{\longmapsto} * e^{\prime \prime \prime}$ and $e^{\prime \prime} \stackrel{n d}{\longrightarrow} e^{\prime \prime \prime}$. Similarly, $d \stackrel{\text { nd }}{\longrightarrow} d^{\prime}$ and $d \stackrel{\text { nd }}{\longmapsto} d^{\prime \prime}$ then there exists a declaration $d^{\prime \prime \prime}$ such that $d^{\prime} \stackrel{n d}{\longmapsto} d^{\prime \prime \prime}$ and $d^{\prime \prime} \stackrel{\text { nd }}{\longmapsto}$ * $d^{\prime \prime \prime}$.

We first prove the following lemma.

Lemma 3. If $e \stackrel{\text { nd }}{\longrightarrow} e^{\prime}$ and $e \stackrel{\text { nd }}{\longmapsto}$ n $e^{\prime \prime}$ then there exists an $e^{\prime \prime \prime}$ such that $e^{\prime} \stackrel{\text { nd }}{\longmapsto}^{*} e^{\prime \prime \prime}$ and $e^{\prime \prime} \stackrel{\text { nd }}{\longmapsto} e^{\prime \prime \prime}$. Similarly, if $d \stackrel{n d}{\longmapsto} d^{\prime}$ and $d \stackrel{n d}{\longrightarrow}{ }^{n} d^{\prime \prime}$ then there exists an $d^{\prime \prime \prime}$ such that $d^{\prime} \stackrel{\text { nd }}{\longrightarrow} d^{\prime \prime \prime}$ and $d^{\prime \prime} \stackrel{n d}{\longmapsto} d^{\prime \prime \prime}$.

Proof. By induction on $n$, using ND-IDLE when $n=0$ and strong confluence in the inductive step. If $n=n^{\prime}+1$ then 
the inductive step can be shown as follows,

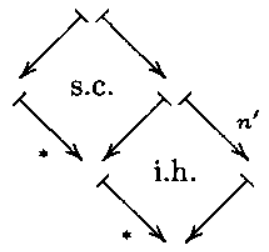

Proof of Theorem 1. Assume $e \stackrel{\text { nd }}{\longmapsto}$ n $e^{\prime}$ and $e \stackrel{\text { nd }}{\longmapsto} e^{\prime \prime}$ (similarly for $d, d^{\prime}, d^{\prime \prime}$ ). Proof by induction on $n$. If $n=0$ then $e=e^{\prime}$. Let $e^{\prime \prime \prime}=e^{\prime \prime}$. We have $e^{\prime} \stackrel{\text { nd }}{\longmapsto}^{*} e^{\prime \prime \prime}$ by the same series of steps taken originally on the left and $e^{\prime \prime} \mapsto^{\text {nd }} \mapsto^{0} e^{\prime \prime \prime}$. If $n>0$ then let $n=n^{\prime}+1$. Applying Lemma 3 and the induction hypothesis yields the desired result as shown below.

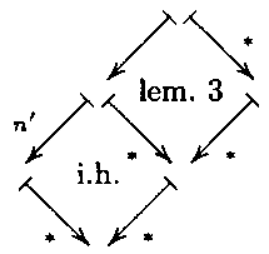

\section{B.2 Completeness}

Theorem 2 (ND Completeness). If $e \Downarrow v$ then $e^{\stackrel{\text { nd }}{\longrightarrow}} v$.

Proof. By induction on the derivation of $e \Downarrow v$.

Case E-FUN: We apply ND-PRIM along with P-FUN to achieve the desired result.

Case E-True: Analogous.

Case E-VAL: This case follows from $v \stackrel{\text { nd }}{\longrightarrow} 00$.

Case E-APP: Applying ND-PRIM along with P-APP, we have let par $x_{1}=e_{1}$ in let par $x_{2}=e_{2}$ in $x_{1} x_{2}$. Inductively, $e_{1} \stackrel{\text { nd }}{\longmapsto}\langle f . x . e\rangle^{\ell}$ and $e_{2} \stackrel{\text { nd }}{\longmapsto} * v_{2}$. We apply rules ND-LET, ND-LEAF, and ND-PRIM to the let par expressions at each step. We obtain the final result by application of P-JOIN and P-APPBETA (along with ND-PRIM in both cases).

Case E-FORK: Applying ND-PRIM along with P-FORK, we have let par $x_{1}=e_{1}$ and $x_{2}=e_{2}$ in $\left\{x_{1}, x_{2}\right\}$. Inductively, $e_{1} \stackrel{\text { nd }}{\longmapsto} * v_{1}$ and $e_{2} \stackrel{\text { nd }}{\longmapsto} v_{2}$. We again apply rules ND-LET, ND-BRANCH, ND-LEAF, and ND-PRIM to the let par expression at each step (also using ND-IDLE in the case where the two sub-computations are of different lengths). We obtain the final result by application of P-JOIN and P-PAIR (along with ND-PRIM in both cases).

Case E-ProJ $i$ : We have \#i $e \stackrel{\text { nd }}{\longmapsto}$ let par $x=e$ in $\# i x$ by rule P-PROJ $i$ with ND-PrIM. Inductively, we have $e \stackrel{\text { nd }}{\longrightarrow} *\left\langle v_{1}, v_{2}\right\rangle^{\ell}$. Applying rules ND-LET, ND-LEAF, and ND-PRIM at each step, we have let par $x=e$ in \#i $x \stackrel{\text { nd }}{\longmapsto}$ * let par $x=\left\langle v_{1}, v_{2}\right\rangle^{\ell}$ in \#ix. We apply rules P-JoIN and P-PROJ ${ }_{i}$ BETA (along with ND-PRIM in both cases) to yield the final result.

Case E-IFTRUE: In this case, we apply ND-PRIM and P-IF. Inductively, we have $e_{1} \stackrel{\text { nd }}{\longmapsto}^{*}$ true $e^{\ell}$. Applying rules ND-LET, ND-LEAF, and ND-PRIM at each step we have let par $x=e_{1}$ in if $x$ then $e_{2}$ else $e_{3} \stackrel{\text { nd }}{\longrightarrow} *$ let par $x=$ true ${ }^{\ell}$ in if $x$ then $e_{2}$ else $e_{3}$. Finally, we apply rules P-JOIN and P-IFTRUE along with ND-PRIM in both steps.

\section{B.3 Soundness}

The following lemma is used in the proof of soundness (Theorem 3).

Lemma 2. If $e \stackrel{\text { nd }}{\longmapsto} e^{\prime}, e^{\prime} \stackrel{\text { nd }}{\longmapsto} v$, and $\left\ulcorner e^{\prime}\right\urcorner \Downarrow v$, then $\left\ulcorner e^{\urcorner} \Downarrow v\right.$. Similarly, if $d \stackrel{\text { nd }}{\longmapsto} d^{\prime}, d^{\prime} \stackrel{\text { nd }}{\longmapsto} \delta$, and $\left\ulcorner d^{\prime}\right\urcorner \Downarrow \delta$, then $\ulcorner d\urcorner \Downarrow \delta$. 
Proof. By induction on the derivations of $e \stackrel{\text { nd }}{\longmapsto} e^{\prime}$ and $d \stackrel{\text { nd }}{\longmapsto} d^{\prime}$.

Case ND-LET: We have $e=$ let par $d$ in $e_{1}$ and $e^{\prime}=$ let par $d^{\prime}$ in $e_{1}$, as well as a derivation of $d \stackrel{\text { nd }}{\longmapsto} d^{\prime}$. Note that $\ulcorner e\urcorner=[\ulcorner d\urcorner]\left\ulcorner e_{1}\right\urcorner$ and $\left\ulcorner e^{\prime}\right\urcorner=\left[\left\ulcorner d^{\prime}\right\urcorner\right]\left\ulcorner e_{1}\right\urcorner$. It is easily shown that $\left[\left\ulcorner d^{\prime}\right\urcorner\right]\left\ulcorner e_{1}\right\urcorner \Downarrow v$ if and only if $\left\ulcorner d^{\prime}\right\urcorner \Downarrow \delta$ and $[\delta]\left\ulcorner e_{1}\right\urcorner \Downarrow v$. Inductively, we have $\ulcorner d\urcorner \Downarrow \delta$. Applying ND-LET yields the desired result.

Case ND-IDLE: In this case, we have $e^{\prime}=e$ and therefore, $\left\ulcorner e^{\prime}\right\urcorner={ }^{\prime} e^{\urcorner}$. The required result is one our assumptions. Case ND-PRIM: We must consider each primitive transition. P-APP, P-PROJ $i$, P-IF, and P-ForK follow immediately since $\left\ulcorner e^{\prime}\right\urcorner=\ulcorner e\urcorner$. In the case of P-JoIN, it is also the true that $\left\ulcorner e^{\prime}\right\urcorner=\ulcorner e\urcorner$. The case for P-Fun follows by application of E-FUN. Similarly for P-True and E-True. For the cases of P-PAIR, P-ProJ ${ }_{i}$ BETA, P-IFTruE, and P-APPBETA, we apply rules E-PAIR, E-PROJ $i$, E-IFTRUE, and E-APP (respectively), using the fact that every value is related to itself in the cost semantics.

Case ND-LeAF: We have $e \stackrel{\text { nd }}{\longmapsto} e^{\prime}$ as a sub-derivation. Inductively, we have $\left\ulcorner e^{\urcorner} \Downarrow v\right.$. Given the form of $d$, it must be the case that $e^{\prime} \stackrel{\text { nd }}{\longmapsto} * v$. The result follows immediately.

Case ND-BRANCH: Here, we have $d_{1} \stackrel{\text { nd }}{\longrightarrow} d_{1}^{\prime}$ and $d_{2} \stackrel{\text { nd }}{\longrightarrow} d_{2}^{\prime}$ as sub-derivations. Inductively, we have $\left.{ }^{r} d_{1}\right\urcorner \Downarrow \delta_{1}$ and $\left\ulcorner d_{2}\right\urcorner \Downarrow \delta_{2}$. Given the form of $d^{\prime}$, it must be the case that $d_{1}^{\prime} \stackrel{\text { nd }}{\longmapsto}{ }^{*} \delta_{1}$ and $d_{2}^{\prime} \stackrel{\text { nd }}{\longmapsto}{ }^{*} \delta_{2}$. The result follows immediately. 